Supporting Information for

\title{
Unimolecular Reactions Following Indoor and Outdoor Limonene Ozonolysis
}

\author{
Jing Chen, ${ }^{\dagger}$ Kristian H. Møller, ${ }^{\dagger}$ Paul O. Wennberg, ${ }^{\ddagger}, \emptyset$ Henrik G. Kjaergaard*,+ \\ ${ }^{\dagger}$ Department of Chemistry, University of Copenhagen, Universitetsparken 5, DK-2100, \\ Copenhagen $\varnothing$, Denmark \\ ${ }^{\ddagger}$ Division of Geological and Planetary Sciences, California Institute of Technology, \\ Pasadena, California 91125, United States \\ II Division of Engineering and Applied Science, California Institute of Technology, \\ Pasadena, California 91125, United States \\ Email: hgk@chem.ku.dk \\ Phone: $+45-35320334$ Fax: $+45-35320322$
}




\section{Contents}

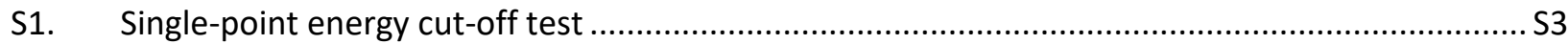

S2. Limonene ozonolysis peroxy radical rate coefficients and related data .....................................S4

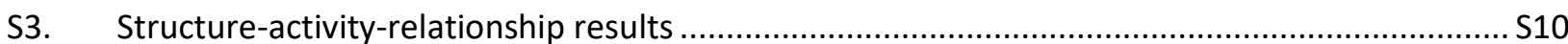

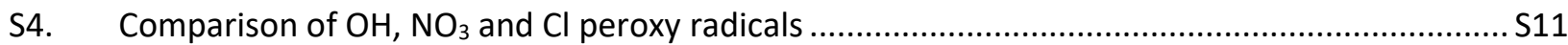

S5. Proposed oxidation pathways in pristine environments .......................................................

S6. Proposed oxidation pathways in polluted environments....................................................... S23

S7. Temperature dependency of the calculated reaction rate coefficients ...................................S27

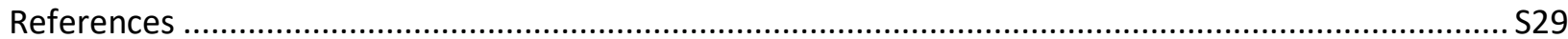

Output files from all calculations including the B3LYP/6-31+G(d) and $\omega B 97 X-D /$ aug-cc-pVTZ xyzgeometries are available online at:

https://sid.erda.dk/public/archives/05c0c3962a7d8c3f01977adb0e694bd1/published-archive.html 


\section{S1. Single-point energy cut-off test}

Due to the large number of conformers for the ring-opened peroxy radicals (typically around 1000 to 4000 conformers for each reactant and TS), a B3LYP single point energy calculation was carried out for the conformers generated by Spartan and only the conformers within a certain energy cut-off were subsequently optimized at the B3LYP level. To find a suitable value for the cut-off, for 17 of the studied reactions, all conformers were optimized at the B3LYP level. For each reactant and transition state, the initial single-point energies and optimized energies were compared to find the single-point energy cutoff needed to retain all the relevant conformers. The data from these tests are given in Table $S 1$. The relevant conformers are defined as those within $2 \mathrm{kcal} / \mathrm{mol}$ in relative electronic energy following the B3LYP optimization. Based on the results in Table S 1, single-point cut-off values of $5 \mathrm{kcal} / \mathrm{mol}$ and 200 $\mathrm{kcal} / \mathrm{mol}$ were chosen for reactants and TSs, respectively.

Table S 1. "Cut-off energy" is the single-point energy cut-off required to retain all relevant conformers. The highest necessary cut-off for any reactant and TS is highlighted in bold. Also the number of relevant conformers and the total number of conformers is given. For each peroxy radical, values for the reactant and all the studied transition states are given.

\begin{tabular}{|c|c|c|c|c|}
\hline Radical & Type & $\begin{array}{l}\text { Cut-off energy } \\
{[\mathrm{kcal} / \mathrm{mol}]}\end{array}$ & \# Relevant conf. & Total \# conf. \\
\hline \multirow{7}{*}{$\mathrm{O}_{\mathrm{O}}$} & Reactant & 4.7 & 29 & 40 \\
\hline & 1,5- $\alpha$-carbonyl & 96.5 & 11 & 23 \\
\hline & 1,6-allylic & 0.9 & 2 & 22 \\
\hline & 1,6-alkyl & 18.5 & 3 & 18 \\
\hline & 1,7-allylic & 48.7 & 3 & 19 \\
\hline & 8-cyclization & 2.5 & 3 & 16 \\
\hline & 1,9-allylic & 39.2 & 3 & 11 \\
\hline \multirow{9}{*}{ L2 } & Reactant & 1.4 & 4 & 15 \\
\hline & 1,4-allylic & 59.1 & 5 & 12 \\
\hline & 1,4-alkyl & 6.9 & 4 & 12 \\
\hline & 1,5-double bond & 0.0 & 1 & 34 \\
\hline & 1,5-allylic & 175.4 & 6 & 7 \\
\hline & 1,5 - $\alpha$-carbonyl & 163.1 & 9 & 11 \\
\hline & 1,7-allylic & 67.7 & 6 & 22 \\
\hline & 5-cyclization & 35.3 & 6 & 12 \\
\hline & 6-cyclization & 2.6 & 3 & 9 \\
\hline \multirow{4}{*}{$\begin{array}{l}0^{\circ}{ }^{\circ} \\
0^{\prime} y^{\prime \prime}\end{array}$} & Reactant & 4.6 & 129 & 730 \\
\hline & 1,6-allylic & 90.4 & 109 & 398 \\
\hline & 1,7-aldehydic & 129.4 & 149 & 480 \\
\hline & 7-cyclization & 198.2 & 315 & 533 \\
\hline
\end{tabular}




\section{S2. Limonene ozonolysis peroxy radical rate coefficients and related data}

Table $S$ 2. The unimolecular reaction rate coefficients for the peroxy radicals $L 1-L 5$ calculated at the $B 3 L Y P / 6-31+G(d), \omega B 97 X-$ $D /$ aug-cc-pVTZ and CCSD(T)-F12a/VDZ-F12 levels using MC-TST. ${ }^{1}$ Only the reactions with a B3LYP level rate coefficient greater than $1 \times 10^{-3} \mathrm{~s}^{-1}$ are calculated at the $\omega B 97 X-D$ and $\operatorname{CCSD}(T)-F 12 a$ levels.

\begin{tabular}{|c|c|c|c|c|}
\hline \multirow{2}{*}{ Radical } & \multirow{2}{*}{ Reaction type } & \multicolumn{3}{|l|}{$\mathrm{k}\left[\mathrm{s}^{-1}\right]$} \\
\hline & & B3LYP & $\omega B 97 X-D$ & $\operatorname{CCSD}(\mathrm{T})-\mathrm{F} 12 \mathrm{a}$ \\
\hline \multirow{8}{*}{$\begin{array}{r}\mathrm{O}_{\mathrm{O}} \\
\mathrm{L1}\end{array}$} & 1,5- $\alpha$-carbonyl & $3.69 \times 10^{-2}$ & $6.81 \times 10^{-2}$ & $1.44 \times 10^{-1}$ \\
\hline & 1,6-allylic & $1.54 \times 10^{1}$ & 4.21 & 3.60 \\
\hline & 1,6-alkyl & $9.83 \times 10^{-3}$ & $4.47 \times 10^{-3}$ & $1.68 \times 10^{-2}$ \\
\hline & 1,7-allylic & 8.70 & 4.49 & 1.21 \\
\hline & 1,7-double bond & $5.95 \times 10^{-15}$ & - & - \\
\hline & 1,9-allylic & $3.87 \times 10^{-6}$ & - & - \\
\hline & 7-cyclization & $6.57 \times 10^{1}$ & 4.71 & 4.59 \\
\hline & 8-cyclization & $2.28 \times 10^{-2}$ & $3.31 \times 10^{-3}$ & $1.15 \times 10^{-2}$ \\
\hline \multirow{8}{*}{ L2 } & 1,4-allylic & $1.22 \times 10^{-2}$ & $2.12 \times 10^{-3}$ & $1.11 \times 10^{-3}$ \\
\hline & 1,4-alkyl & $1.35 \times 10^{-8}$ & - & - \\
\hline & 1,5-double bond & $2.78 \times 10^{-19}$ & - & - \\
\hline & 1,5-allylic & $1.11 \times 10^{-1}$ & $1.76 \times 10^{-2}$ & $4.55 \times 10^{-2}$ \\
\hline & 1,5- $\alpha$-carbonyl & $4.90 \times 10^{-6}$ & - & - \\
\hline & 1,7-allylic & $2.97 \times 10^{-12}$ & - & - \\
\hline & 5-cyclization & $6.03 \times 10^{-1}$ & $2.26 \times 10^{-3}$ & $7.58 \times 10^{-3}$ \\
\hline & 6-cyclization & $3.17 \times 10^{-3}$ & $6.29 \times 10^{-5}$ & $2.10 \times 10^{-3}$ \\
\hline \multirow{7}{*}{$\underbrace{\substack{\vdots \\
\vdots}}_{(R, R)-\mathrm{L}}$} & 1,5- $\alpha$-carbonyl & $3.56 \times 10^{-6}$ & - & - \\
\hline & 1,5-allylic & $5.58 \times 10^{-1}$ & $9.37 \times 10^{-2}$ & $2.68 \times 10^{-1}$ \\
\hline & 1,6- $\alpha$-carbonyl & $4.66 \times 10^{-1}$ & $5.73 \times 10^{-2}$ & $7.20 \times 10^{-2}$ \\
\hline & 1,7-aldehydic & $4.25 \times 10^{2}$ & $2.53 \times 10^{1}$ & 4.16 \\
\hline & 1,7-allylic & 2.99 & 1.41 & $7.88 \times 10^{-1}$ \\
\hline & 6-cyclization & $1.38 \times 10^{1}$ & $4.04 \times 10^{-1}$ & 3.86 \\
\hline & 7-cyclization & $2.37 \times 10^{1}$ & $4.37 \times 10^{-1}$ & $6.61 \times 10^{-1}$ \\
\hline \multirow{7}{*}{ 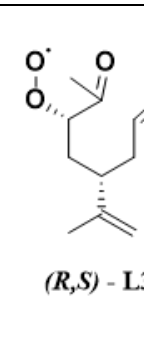 } & 1,5- $\alpha$-carbonyl & $2.84 \times 10^{-6}$ & - & - \\
\hline & 1,5-allylic & 2.24 & $1.65 \times 10^{-1}$ & $5.66 \times 10^{-1}$ \\
\hline & 1,6-a-carbonyl & $5.88 \times 10^{-2}$ & $1.22 \times 10^{-2}$ & $1.80 \times 10^{-2}$ \\
\hline & 1,7-aldehydic & $2.79 \times 10^{2}$ & $1.27 \times 10^{1}$ & 6.69 \\
\hline & 1,7-allylic & $2.46 \times 10^{1}$ & 3.66 & 4.35 \\
\hline & 6-cyclization & $6.27 \times 10^{1}$ & $4.05 \times 10^{-1}$ & 2.98 \\
\hline & 7-cyclization & $1.15 \times 10^{2}$ & $5.91 \times 10^{-1}$ & $3.23 \times 10^{-1}$ \\
\hline \multirow{7}{*}{$\underbrace{=}_{\text {L4 }}$} & 1,5 - $\alpha$-carbonyl & $4.37 \times 10^{-4}$ & - & - \\
\hline & 1,7-allylic & $6.44 \times 10^{-1}$ & $6.96 \times 10^{-1}$ & $6.63 \times 10^{-1}$ \\
\hline & 1,8-a-carbonyl & $5.13 \times 10^{-3}$ & $2.26 \times 10^{-3}$ & $1.54 \times 10^{-3}$ \\
\hline & 1,9-aldehydic & $2.09 \times 10^{1}$ & 9.13 & $4.50 \times 10^{-1}$ \\
\hline & 1,9-allylic & $1.65 \times 10^{-1}$ & $7.85 \times 10^{-2}$ & $3.02 \times 10^{-2}$ \\
\hline & 8-cyclization & $1.61 \times 10^{-5}$ & - & - \\
\hline & 9-cyclization & 1.32 & $5.72 \times 10^{-2}$ & $3.50 \times 10^{-2}$ \\
\hline
\end{tabular}




\begin{tabular}{|c|c|c|c|c|}
\hline \multirow{8}{*}{$\underbrace{O}_{(S, S)-L 5}$} & 1,4-allylic & $3.48 \times 10^{-5}$ & - & - \\
\hline & 1,4-aldehydic & $1.50 \times 10^{-1}$ & $2.17 \times 10^{-1}$ & 0.60 \\
\hline & 1,5-alkyl & $3.31 \times 10^{-4}$ & $2.58 \times 10^{-5}$ & $3.48 \times 10^{-4}$ \\
\hline & 1,6-allylic & $1.58 \times 10^{-1}$ & $3.53 \times 10^{-2}$ & $3.28 \times 10^{-2}$ \\
\hline & 1,6 - $\alpha$-carbonyl & $1.98 \times 10^{-2}$ & $1.26 \times 10^{-2}$ & $1.18 \times 10^{-2}$ \\
\hline & 1,8 - $\alpha$-carbonyl & $1.01 \times 10^{-6}$ & - & - \\
\hline & 5-cyclization & $2.69 \times 10^{3}$ & $3.86 \times 10^{1}$ & $2.27 \times 10^{2}$ \\
\hline & 6-cyclization & $6.36 \times 10^{2}$ & 3.66 & $1.99 \times 10^{1}$ \\
\hline \multirow{8}{*}{$\underbrace{O}_{(R, S)-\mathrm{L} 5}$} & 1,4-allylic & $1.36 \times 10^{-5}$ & - & - \\
\hline & 1,4-aldehydic & $4.63 \times 10^{-1}$ & $2.07 \times 10^{-1}$ & $4.91 \times 10^{-1}$ \\
\hline & 1,5-alkyl & $6.16 \times 10^{-3}$ & $1.50 \times 10^{-3}$ & $9.28 \times 10^{-3}$ \\
\hline & 1,6-allylic & $1.38 \times 10^{-1}$ & $3.78 \times 10^{-2}$ & $2.78 \times 10^{-2}$ \\
\hline & 1,6-a-carbonyl & $1.07 \times 10^{-1}$ & $4.87 \times 10^{-2}$ & $6.43 \times 10^{-2}$ \\
\hline & 1,8- $\alpha$-carbonyl & $6.42 \times 10^{-6}$ & - & - \\
\hline & 5-cyclization & $4.86 \times 10^{2}$ & $1.56 \times 10^{1}$ & $4.47 \times 10^{1}$ \\
\hline & 6-cyclization & $5.38 \times 10^{1}$ & $8.97 \times 10^{-1}$ & 1.35 \\
\hline
\end{tabular}


Table S 3. Calculated B3LYP level reaction rate coefficients $(k)$, reaction energy barriers $\left(E_{a}\right)$, tunneling factors $(k)$, imaginary frequencies ( $\tilde{V})$ and ratios of summed partition functions exponentially weighted by relative conformer energy $\left(Q_{T S} / Q_{R}\right)$ for all seven peroxy radicals (L1-L5). A symmetric reaction barrier $\left(E_{T S}-E_{R}=E_{T S}-E_{P}\right)$ was assumed when calculating the B3LYP level tunneling factors (see method section in main text).

\begin{tabular}{|c|c|c|c|c|c|c|}
\hline Radical & Reaction type & $\mathrm{k}\left[\mathrm{s}^{-1}\right]$ & $\mathrm{E}_{\mathrm{a}}[\mathrm{kcal} / \mathrm{mol}]$ & $\mathrm{K}$ & $\tilde{\mathrm{v}}\left[\mathrm{cm}^{-1}\right]$ & $\mathrm{Q}_{\mathrm{TS}} / \mathrm{Q}_{\mathrm{R}}$ \\
\hline \multirow{8}{*}{ Ll } & 1,5- $\alpha$-carbonyl & $3.7 \times 10^{-2}$ & 20.7 & 325.2 & 1842.2 & 0.0271 \\
\hline & 1,6-allylic & $1.5 \times 10^{1}$ & 16.1 & 140.9 & 1832.5 & 0.0113 \\
\hline & 1,6-alkyl & $9.8 \times 10^{-3}$ & 20.1 & 69.1 & 1636.4 & 0.0126 \\
\hline & 1,7-allylic & 8.7 & 16.2 & 65.9 & 1698.9 & 0.0154 \\
\hline & 1,7-double bond & $6.0 \times 10^{-15}$ & 38.4 & 1140.1 & 1731.0 & 0.0115 \\
\hline & 1,9-allylic & $3.9 \times 10^{-6}$ & 25.1 & 318.0 & 1758.7 & 0.0052 \\
\hline & 7-cyclization & $6.6 \times 10^{1}$ & 12.3 & 1.2 & 443.4 & 0.0090 \\
\hline & 8-cyclization & $2.3 \times 10^{-2}$ & 16.8 & 1.2 & 427.4 & 0.0060 \\
\hline \multirow{8}{*}{ L2 } & 1,4-allylic & $1.2 \times 10^{-2}$ & 23.4 & 1287.5 & 1951.3 & 0.2147 \\
\hline & 1,4-alkyl & $1.4 \times 10^{-8}$ & 34.0 & 37169.4 & 2051.4 & 0.5065 \\
\hline & 1,5-double bond & $2.8 \times 10^{-19}$ & 51.5 & 4194702.5 & 2139.9 & 0.5680 \\
\hline & 1,5-allylic & $1.1 \times 10^{-1}$ & 21.2 & 350.2 & 1841.1 & 0.1745 \\
\hline & 1,5- $\alpha$-carbonyl & $4.9 \times 10^{-6}$ & 28.1 & 2504.7 & 1922.8 & 0.1308 \\
\hline & 1,7-allylic & $3.0 \times 10^{-12}$ & 36.7 & 6887.1 & 1881.7 & 0.0570 \\
\hline & 5-cyclization & $6.0 \times 10^{-1}$ & 17.0 & 1.3 & 526.4 & 0.2096 \\
\hline & 6-cyclization & $3.2 \times 10^{-3}$ & 19.7 & 1.3 & 489.6 & 0.1032 \\
\hline \multirow{7}{*}{$\underbrace{\substack{0 \\
\text { i. }}}_{(R, R)-\mathrm{L} 3}$} & 1,5- $\alpha$-carbonyl & $3.6 \times 10^{-6}$ & 28.5 & 2626.8 & 1921.4 & 0.1593 \\
\hline & 1,5-allylic & $5.6 \times 10^{-1}$ & 18.3 & 39.2 & 1572.0 & 0.0620 \\
\hline & 1,6-a-carbonyl & $4.7 \times 10^{-1}$ & 19.1 & 220.9 & 1824.2 & 0.0359 \\
\hline & 1,7-aldehydic & $4.3 \times 10^{2}$ & 14.0 & 31.7 & 1606.6 & 0.0384 \\
\hline & 1,7-allylic & 3.0 & 17.2 & 88.8 & 1727.3 & 0.0221 \\
\hline & 6-cyclization & $1.4 \times 10^{1}$ & 13.2 & 1.2 & 443.6 & 0.0085 \\
\hline & 7-cyclization & $2.4 \times 10^{1}$ & 14.1 & 1.2 & 439.1 & 0.0642 \\
\hline \multirow{7}{*}{$\begin{array}{l}0 \\
0 \\
0, \ldots\end{array}$} & 1,5- $\alpha$-carbonyl & $2.8 \times 10^{-6}$ & 28.0 & 2390.5 & 1920.2 & 0.0672 \\
\hline & 1,5-allylic & 2.2 & 17.9 & 91.6 & 1717.0 & 0.0547 \\
\hline & 1,6- $\alpha$-carbonyl & $5.9 \times 10^{-2}$ & 19.1 & 214.4 & 1819.6 & 0.0048 \\
\hline & 1,7-aldehydic & $2.8 \times 10^{2}$ & 12.9 & 29.0 & 1612.2 & 0.0042 \\
\hline & 1,7-allylic & $2.5 \times 10^{1}$ & 14.8 & 72.6 & 1749.8 & 0.0039 \\
\hline & 6-cyclization & $6.3 \times 10^{1}$ & 12.0 & 1.2 & 461.7 & 0.0050 \\
\hline & 7-cyclization & $1.1 \times 10^{2}$ & 12.7 & 1.2 & 451.8 & 0.0285 \\
\hline \multirow{7}{*}{$\underbrace{\sum_{1}^{0}}_{1}$} & 1,5- $\alpha$-carbonyl & $4.4 \times 10^{-4}$ & 24.4 & 1193.1 & 1981.9 & 0.0486 \\
\hline & 1,7-allylic & $6.4 \times 10^{-1}$ & 17.2 & 50.5 & 1633.0 & 0.0080 \\
\hline & 1,8- $\alpha$-carbonyl & $5.1 \times 10^{-3}$ & 20.7 & 373.0 & 1859.5 & 0.0035 \\
\hline & 1,9-aldehydic & $2.1 \times 10^{1}$ & 14.4 & 42.4 & 1656.0 & 0.0029 \\
\hline & 1,9-allylic & $1.7 \times 10^{-1}$ & 19.0 & 299.3 & 1872.6 & 0.0070 \\
\hline & 8-cyclization & $1.6 \times 10^{-5}$ & 19.8 & 1.2 & 447.3 & 0.0007 \\
\hline & 9-cyclization & 1.3 & 13.7 & 1.3 & 486.9 & 0.0017 \\
\hline
\end{tabular}




\begin{tabular}{|c|c|c|c|c|c|c|}
\hline \multirow{8}{*}{$\underbrace{11}_{(S, S)-L}$} & 1,4-allylic & $3.5 \times 10^{-5}$ & 26.8 & 5440.6 & 2026.4 & 0.0491 \\
\hline & 1,4-aldehydic & $1.5 \times 10^{-1}$ & 20.7 & 1809.9 & 255.2 & 0.1383 \\
\hline & 1,5-alkyl & $3.3 \times 10^{-4}$ & 23.5 & 93.7 & 1634.5 & 0.0893 \\
\hline & 1,6-allylic & $1.6 \times 10^{-1}$ & 19.8 & 454.5 & 1910.9 & 0.0170 \\
\hline & 1,6 - $\alpha$-carbonyl & $2.0 \times 10^{-2}$ & 20.4 & 420.7 & 1833.9 & 0.0068 \\
\hline & 1,8- $\alpha$-carbonyl & $1.0 \times 10^{-6}$ & 25.7 & 250.2 & 1723.3 & 0.0048 \\
\hline & 5-cyclization & $2.7 \times 10^{3}$ & 11.2 & 1.2 & 445.7 & 0.0577 \\
\hline & 6-cyclization & $6.4 \times 10^{2}$ & 11.8 & 1.3 & 500.5 & 0.0364 \\
\hline \multirow{8}{*}{$\underbrace{O}_{(R, S)}$} & 1,4-allylic & $1.4 \times 10^{-5}$ & 27.8 & 6059.5 & 2015.3 & 0.0863 \\
\hline & 1,4-aldehydic & $4.6 \times 10^{-1}$ & 20.9 & 306.8 & 1830.2 & 0.5001 \\
\hline & 1,5-alkyl & $6.2 \times 10^{-3}$ & 21.7 & 85.3 & 1644.3 & 0.0952 \\
\hline & 1,6-allylic & $1.4 \times 10^{-1}$ & 20.0 & 479.0 & 1910.7 & 0.0226 \\
\hline & 1,6- $\alpha$-carbonyl & $1.1 \times 10^{-1}$ & 20.1 & 255.7 & 1822.0 & 0.0386 \\
\hline & 1,8- $\alpha$-carbonyl & $6.4 \times 10^{-6}$ & 24.5 & 233.5 & 1733.3 & 0.0037 \\
\hline & 5-cyclization & $4.9 \times 10^{2}$ & 11.7 & 1.2 & 460.2 & 0.0221 \\
\hline & 6-cyclization & $5.4 \times 10^{1}$ & 13.5 & 1.3 & 510.5 & 0.0516 \\
\hline
\end{tabular}


Table S 4. Calculated $\omega B 97 X-D$ level reaction rate coefficients $\left(k, s^{-1}\right)$, reaction energy barriers $\left(E_{a}, k c a l / m o l\right)$, tunneling factors $(k)$, imaginary frequencies $\left(\tilde{v}, \mathrm{~cm}^{-1}\right)$ and ratios of summed partition functions exponentially weighted by relative conformer energy $\left(Q_{T S} / Q_{R}\right)$, as well as the calculated CCSD $(T)$-F12a level reaction rate coefficients $\left(k, s^{-1}\right)$, reaction energy barriers $\left(E_{a}, k c a l / m o l\right)$ and tunneling factors ( $\mathrm{K}$ ). For four of the allylic $\mathrm{H}$-shifts from the methyl group (indicated by a dash), the IRC end points at the CCSD(T)F12a level had convergence issues and the $\omega B 97 X-D$ level tunneling factors were used instead.

\begin{tabular}{|c|c|c|c|c|c|c|c|c|c|}
\hline \multirow{2}{*}{ Radical } & \multirow{2}{*}{ type } & \multicolumn{5}{|l|}{$\omega B 97 X-D$} & \multicolumn{3}{|c|}{$\operatorname{CCSD}(T)-F 12 a$} \\
\hline & & k & $\mathrm{E}_{\mathrm{a}}$ & K & $\tilde{v}$ & $\mathrm{Q}_{\mathrm{TS}} / \mathrm{Q}_{\mathrm{R}}$ & k & $\mathrm{E}_{\mathrm{a}}$ & K \\
\hline \multirow{6}{*}{$\begin{array}{r}\mathrm{O}^{\circ} \\
\mathrm{Ll}\end{array}$} & 1,5 - $\alpha$-carbonyl & $6.8 \times 10^{-2}$ & 21.2 & 679.5 & 1993.7 & 0.0549 & $1.4 \times 10^{-1}$ & 20.8 & 718.2 \\
\hline & 1,6-allylic & 4.2 & 18.1 & 578.0 & 2003.3 & 0.0231 & 3.6 & 18.3 & 639.9 \\
\hline & 1,6-alkyl & $4.5 \times 10^{-3}$ & 20.4 & 26.4 & 1940.9 & 0.0257 & $1.7 \times 10^{-2}$ & 20.0 & 50.6 \\
\hline & 1,7-allylic & 4.5 & 17.1 & 79.4 & 1800.8 & 0.0308 & 1.2 & 18.0 & 106.6 \\
\hline & 7-cyclization & 4.7 & 14.5 & 1.3 & 519.9 & 0.0233 & 4.6 & 14.5 & 1.3 \\
\hline & 8-cyclization & $3.3 \times 10^{-3}$ & 18.6 & 1.3 & 522.5 & 0.0164 & $1.1 \times 10^{-2}$ & 17.8 & 1.3 \\
\hline \multirow{4}{*}{ L2 } & 1,4-allylic & $2.1 \times 10^{-3}$ & 26.0 & 16073.9 & 2167.2 & 0.2301 & $1.1 \times 10^{-3}$ & 26.6 & 25272.5 \\
\hline & 1,5-allylic & $1.8 \times 10^{-2}$ & 22.7 & 688.2 & 1979.0 & 0.1834 & $4.5 \times 10^{-2}$ & 22.2 & 801.4 \\
\hline & 5-cyclization & $2.3 \times 10^{-3}$ & 20.3 & 1.5 & -633.6 & 0.1957 & $7.6 \times 10^{-3}$ & 19.8 & 1.5 \\
\hline & 6-cyclization & $6.3 \times 10^{-5}$ & 22.6 & 1.4 & -585.6 & 0.2635 & $2.1 \times 10^{-3}$ & 20.5 & 1.4 \\
\hline \multirow{6}{*}{$\underbrace{1}_{(R, R)-L}$} & 1,5-allylic & $9.4 \times 10^{-2}$ & 19.1 & 52.6 & 1702.0 & 0.0300 & $2.7 \times 10^{-1}$ & 18.5 & 76.1 \\
\hline & 1,6 - $\alpha$-carbonyl & $5.7 \times 10^{-2}$ & 21.5 & 826.7 & 2065.9 & 0.0634 & $7.2 \times 10^{-2}$ & 21.6 & 1155.5 \\
\hline & 1,7-aldehydic & $2.5 \times 10^{1}$ & 15.3 & 22.2 & 1643.4 & 0.0326 & 4.2 & 16.6 & 30.5 \\
\hline & 1,7-allylic & 1.4 & 17.9 & 131.9 & 1906.4 & 0.0236 & $7.9 \times 10^{-1}$ & 18.3 & - \\
\hline & 6-cyclization & $4.0 \times 10^{-1}$ & 15.4 & 1.4 & 559.0 & 0.0092 & 3.9 & 14.1 & 1.4 \\
\hline & 7-cyclization & $4.4 \times 10^{-1}$ & 16.7 & 1.3 & 515.8 & 0.0903 & $6.6 \times 10^{-1}$ & 16.4 & 1.3 \\
\hline \multirow{6}{*}{ (R,S) - L } & 1,5-allylic & $1.7 \times 10^{-1}$ & 19.7 & 78.6 & 1727.3 & 0.0945 & $5.7 \times 10^{-1}$ & 19.0 & 78.3 \\
\hline & 1,6 - $\alpha$-carbonyl & $1.2 \times 10^{-2}$ & 21.1 & 245.9 & 2054.2 & 0.0249 & $1.8 \times 10^{-2}$ & 21.1 & 339.6 \\
\hline & 1,7-aldehydic & $1.3 \times 10^{1}$ & 15.6 & 28.5 & 1638.8 & 0.0201 & 6.7 & 15.7 & 36.4 \\
\hline & 1,7-allylic & 3.7 & 17.0 & 170.1 & 1935.5 & 0.0108 & 4.4 & 16.9 & - \\
\hline & 6-cyclization & $4.0 \times 10^{-1}$ & 15.7 & 1.4 & 579.3 & 0.0138 & 3.0 & 15.1 & 1.4 \\
\hline & 7-cyclization & $5.9 \times 10^{-1}$ & 16.2 & 1.4 & 542.7 & 0.0516 & $3.2 \times 10^{-1}$ & 16.5 & 1.4 \\
\hline
\end{tabular}




\begin{tabular}{|c|c|c|c|c|c|c|c|c|c|}
\hline \multirow{5}{*}{ o. } & 1,7-allylic & $7.0 \times 10^{-1}$ & 18.1 & 158.3 & 1851.8 & 0.0131 & $6.6 \times 10^{-1}$ & 18.2 & 175.0 \\
\hline & $1,8-\alpha$-carbonyl & $2.3 \times 10^{-3}$ & 21.3 & 481.9 & 2114.3 & 0.0032 & $1.5 \times 10^{-3}$ & 21.9 & 834.7 \\
\hline & 1,9-aldehydic & 9.1 & 14.9 & 35.5 & 1750.2 & 0.0033 & $4.5 \times 10^{-1}$ & 17.0 & 61.4 \\
\hline & 1,9-allylic & $7.9 \times 10^{-2}$ & 19.6 & 674.0 & 2086.8 & 0.0045 & $3.0 \times 10^{-2}$ & 20.2 & 674.0 \\
\hline & 9-cyclization & $5.7 \times 10^{-2}$ & 15.9 & 1.5 & 601.3 & 0.0029 & $3.5 \times 10^{-2}$ & 16.2 & 1.46 \\
\hline \multirow{6}{*}{$\underbrace{1}_{(S, S)-L 5}$} & 1,4-aldehydic & $3.4 \times 10^{-1}$ & 20.5 & 158.7 & 1813.4 & 0.3323 & 0.6 & 20.0 & 209.4 \\
\hline & 1,5-alkyl & $2.6 \times 10^{-5}$ & 24.6 & 35.0 & 1815.7 & 0.1293 & $3.5 \times 10^{-4}$ & 11.7 & 42.4 \\
\hline & 1,6-allylic & $3.5 \times 10^{-2}$ & 21.1 & 596.0 & 2063.8 & 0.0263 & $3.3 \times 10^{-2}$ & 21.1 & - \\
\hline & 1,6- $\alpha$-carbonyl & $1.3 \times 10^{-2}$ & 21.6 & 773.7 & 2052.7 & 0.0171 & $1.2 \times 10^{-2}$ & 21.8 & 1107.9 \\
\hline & 5-cyclization & $3.9 \times 10^{1}$ & 13.9 & 1.4 & 550.1 & 0.0683 & $2.3 \times 10^{2}$ & 12.8 & 1.4 \\
\hline & 6-cyclization & 3.7 & 15.1 & 1.4 & 578.9 & 0.0293 & $2.0 \times 10^{1}$ & 14.1 & 1.4 \\
\hline \multirow{6}{*}{ 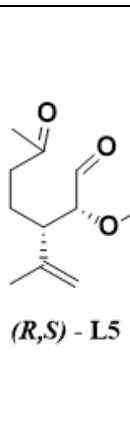 } & 1,4-aldehydic & $2.1 \times 10^{-1}$ & 20.6 & 133.4 & 1797.8 & 0.3112 & $4.9 \times 10^{-1}$ & 20.2 & 173.4 \\
\hline & 1,5-alkyl & $1.5 \times 10^{-3}$ & 21.8 & 25.9 & 1804.8 & 0.0937 & $9.3 \times 10^{-3}$ & 21.0 & 39.3 \\
\hline & 1,6-allylic & $7.0 \times 10^{-2}$ & 21.0 & 1105.4 & 2063.8 & 0.0254 & $2.8 \times 10^{-2}$ & 21.2 & - \\
\hline & 1,6- $\alpha$-carbonyl & $4.9 \times 10^{-2}$ & 21.1 & 438.9 & 2039.4 & 0.0537 & $6.4 \times 10^{-2}$ & 21.1 & 608.3 \\
\hline & 5-cyclization & $1.6 \times 10^{1}$ & 13.9 & 1.4 & 587.5 & 0.0273 & $4.5 \times 10^{1}$ & 13.3 & 1.4 \\
\hline & 6-cyclization & $9.0 \times 10^{-1}$ & 16.3 & 1.4 & 570.6 & 0.0848 & 1.4 & 16.0 & 1.4 \\
\hline
\end{tabular}




\section{S3. Structure-activity-relationship results}

Table S 5. Calculated $\mathrm{H}$-shift reaction rate coefficients for the $\mathrm{O}_{3}$ initiated peroxy radicals $\mathrm{L} 1$ - $L 5$ for limonene compared to the predictions from the available structure-activity-relationship $(S A R)^{2}$. Reactions for $L 1$ and $L 2$ are shown in gray since the SAR does not include effect of rings but $L 1$ and $L 2$ have six-membered rings in their structures. The SAR is only available for the 1,4-1,7 $H$ shifts.

\begin{tabular}{|c|c|c|c|c|}
\hline \multirow{2}{*}{ Radical } & \multirow{2}{*}{ Reaction type } & \multicolumn{2}{|l|}{$\mathrm{k}\left[\mathrm{s}^{-1}\right]$} & \multirow[b]{2}{*}{$\mathrm{k}_{\text {calc. }} / \mathrm{k}_{\mathrm{SAR}}$} \\
\hline & & Calculated & $\mathrm{SAR}^{\mathrm{a}}$ & \\
\hline \multirow{4}{*}{ L1 } & 1,5 - $\alpha$-carbonyl & $1.4 \times 10^{-1}$ & $7.70 \times 10^{-3}$ & 18.80 \\
\hline & 1,6-allylic & 3.6 & 2.04 & 1.76 \\
\hline & 1,6-alkyl & $1.7 \times 10^{-2}$ & $8.84 \times 10^{-4}$ & 19.03 \\
\hline & 1,7-allylic & 1.2 & 8.22 & 0.15 \\
\hline \multirow{2}{*}{ L2 } & 1,4-allylic & $1.1 \times 10^{-3}$ & $2.55 \times 10^{-5}$ & 43.43 \\
\hline & 1,5-allylic & $4.5 \times 10^{-2}$ & $4.60 \times 10^{-5}$ & 988.08 \\
\hline \multirow{4}{*}{$(R, R)-\mathrm{L} 3$} & 1,5 -allylic & $3.8 \times 10^{-1}$ & 1.87 & 0.21 \\
\hline & 1,6- $\alpha$-carbonyl & $7.2 \times 10^{-2}$ & $7.83 \times 10^{-3}$ & 9.20 \\
\hline & 1,7-aldehydic & 4.2 & $1.71 \times 10^{-1}$ & 24.34 \\
\hline & 1,7-allylic & $7.9 \times 10^{-1}$ & $3.72 \times 10^{-1}$ & 2.12 \\
\hline \multirow{4}{*}{$(R, S)-\mathrm{L} 3$} & 1,5 -allylic & $5.7 \times 10^{-1}$ & 1.87 & 0.30 \\
\hline & $1,6-\alpha$-carbonyl & $1.8 \times 10^{-2}$ & $7.83 \times 10^{-3}$ & 2.30 \\
\hline & 1,7-aldehydic & 6.7 & $1.71 \times 10^{-1}$ & 39.18 \\
\hline & 1,7-allylic & 4.4 & $3.72 \times 10^{-1}$ & 11.70 \\
\hline \multirow{4}{*}{ L4 } & 1,7-allylic & $6.6 \times 10^{-1}$ & $2.55 \times 10^{-2}$ & 26.01 \\
\hline & 1,8 - $\alpha$-carbonyl & $1.5 \times 10^{-3}$ & - & - \\
\hline & 1,9-aldehydic & $4.5 \times 10^{-1}$ & - & - \\
\hline & 1,9-allylic & $3.0 \times 10^{-2}$ & - & - \\
\hline \multirow{4}{*}{$(S, S)-\mathrm{L} 5$} & 1,4-aldehydic & 0.6 & $6.64 \times 10^{-2}$ & 1.1 \\
\hline & 1,5-alkyl & $3.5 \times 10^{-4}$ & $1.23 \times 10^{-3}$ & 0.28 \\
\hline & 1,6-allylic & $3.3 \times 10^{-2}$ & $1.55 \times 10^{-2}$ & 2.11 \\
\hline & 1,6 - $\alpha$-carbonyl & $1.2 \times 10^{-2}$ & $7.83 \times 10^{-3}$ & 1.51 \\
\hline \multirow{4}{*}{$(R, S)-\mathrm{L} 5$} & 1,4-aldehydic & $4.9 \times 10^{-1}$ & $6.64 \times 10^{-2}$ & 7.39 \\
\hline & 1,5 -alkyl & $9.3 \times 10^{-3}$ & $1.23 \times 10^{-3}$ & 7.55 \\
\hline & 1,6-allylic & $2.8 \times 10^{-2}$ & $1.55 \times 10^{-2}$ & 1.81 \\
\hline & 1,6- $\alpha$-carbonyl & $6.4 \times 10^{-2}$ & $7.83 \times 10^{-3}$ & 8.22 \\
\hline
\end{tabular}

a. Calculated using the SAR in ref. ${ }^{2}$ 


\section{S4. Comparison of $\mathrm{OH}, \mathrm{NO}_{3}$ and $\mathrm{Cl}$ peroxy radicals}

Table S 6. B3LYP reaction rate coefficients $(k)$, energy barriers $\left(E_{a}\right)$, tunneling factors $(k)$ and ratios of summed partition functions exponentially weighted by relative conformer energy $\left(Q_{T S} / Q_{R}\right)$ of the $\mathrm{OH}, \mathrm{NO}_{3}$ and $\mathrm{Cl}$ initiated peroxy radicals (La and $\left.\mathrm{Lb}\right)$. The functional group (R) can be either $\mathrm{OH}, \mathrm{NO}_{3}$ or $\mathrm{Cl}$.

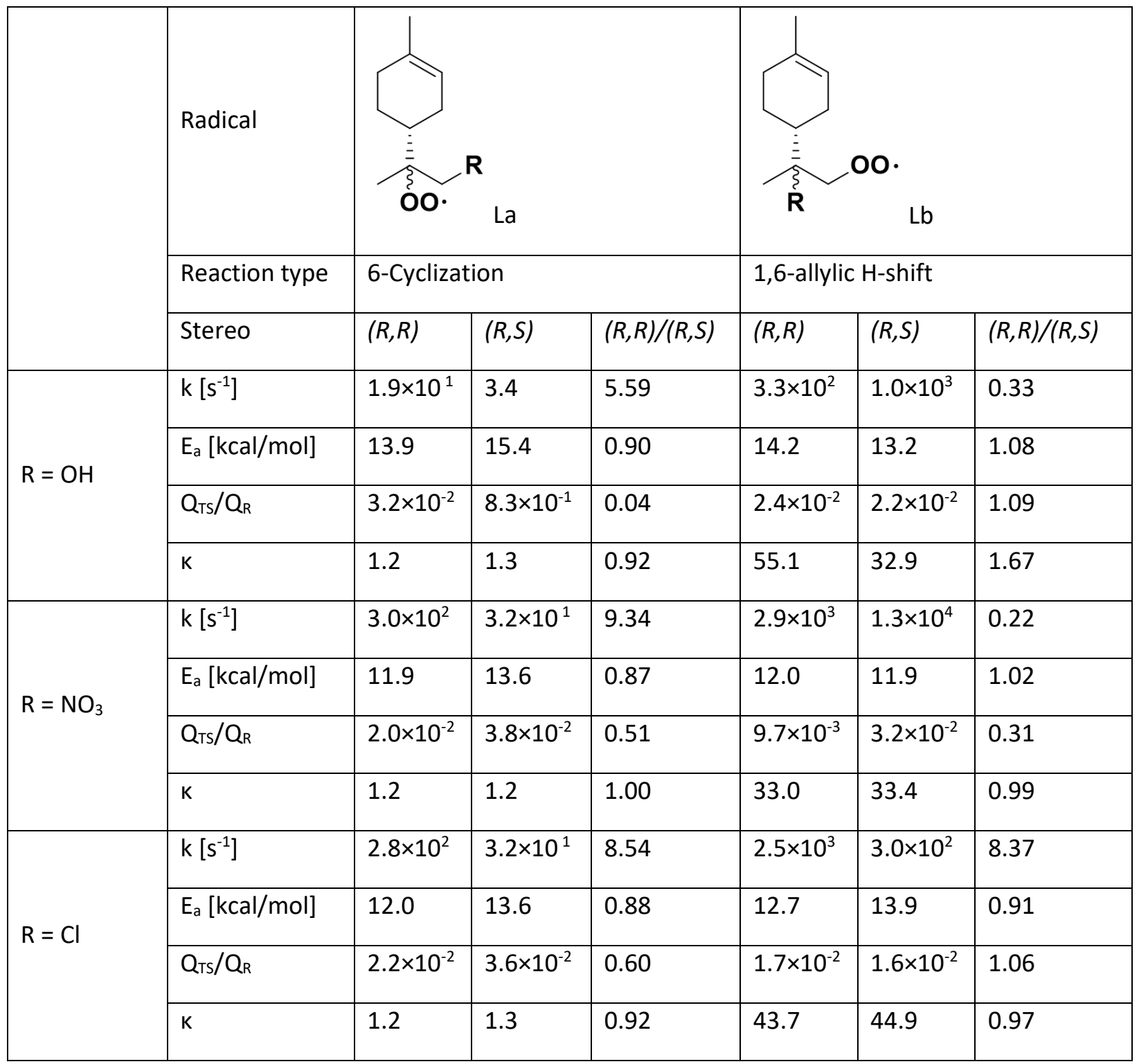




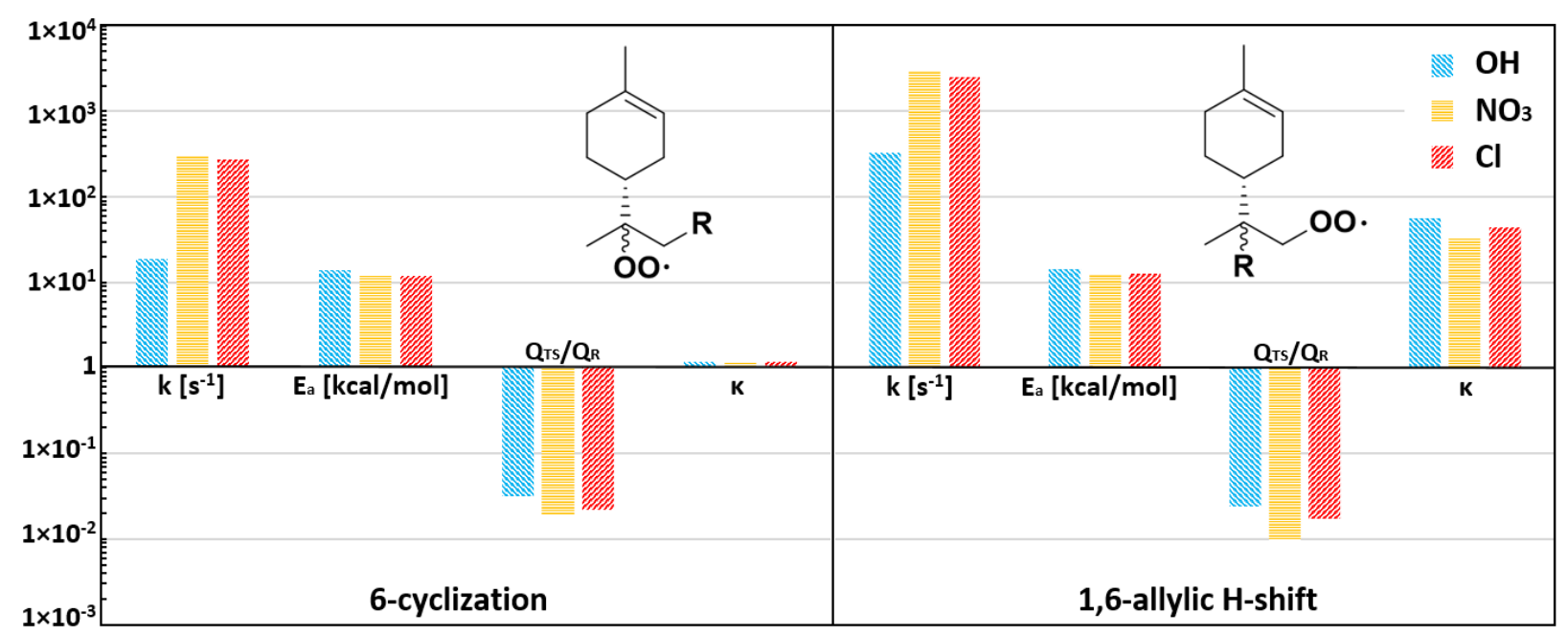

Figure $S$ 1. Comparison of the unimolecular reaction rate coefficients $(k)$, reaction energy barriers $\left(E_{a}\right)$, ratios of summed partition functions exponentially weighted by relative conformer energy $\left(Q_{T S} / Q_{R}\right)$ and tunneling factors $(K)$ between $\mathrm{OH}, \mathrm{NO} \mathrm{O}_{3}$ and $\mathrm{Cl}$ peroxy radical ( $L a$ and $L b$ ) calculated at the B3LYP level. The functional group $R$ can be either $\mathrm{OH}, \mathrm{NO}_{3}$ or $C l$. Only the results for the ( $R, R$ ) stereoisomers are shown here. The corresponding values for the $(R, S)$ stereoisomers can be found in Table $S 6$. 
Table $S$ 7. $\omega B 97 X-D$ reaction rate coefficients $(k)$, energy barriers $\left(E_{a}\right)$, tunneling factors $(k)$ and and ratios of summed partition functions exponentially weighted by relative conformer energy $\left(Q_{T S} / Q_{R}\right)$ of the $\mathrm{OH}, \mathrm{NO}_{3}$ and $\mathrm{Cl}$ initiated peroxy radicals (La and $\mathrm{Lb}$ ). The functional group (R) can be either $\mathrm{OH}, \mathrm{NO}_{3}$ or $\mathrm{Cl}$.

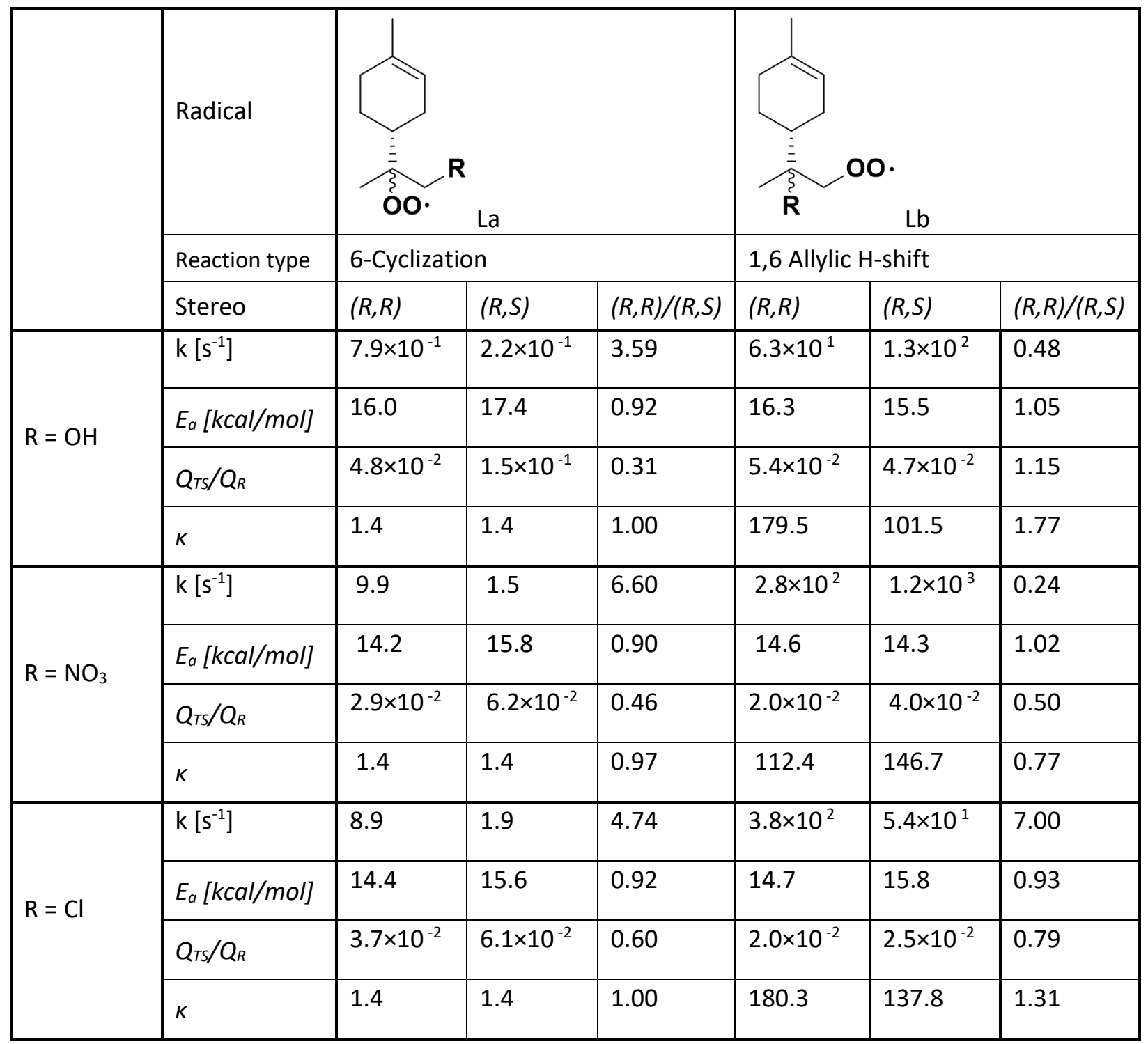




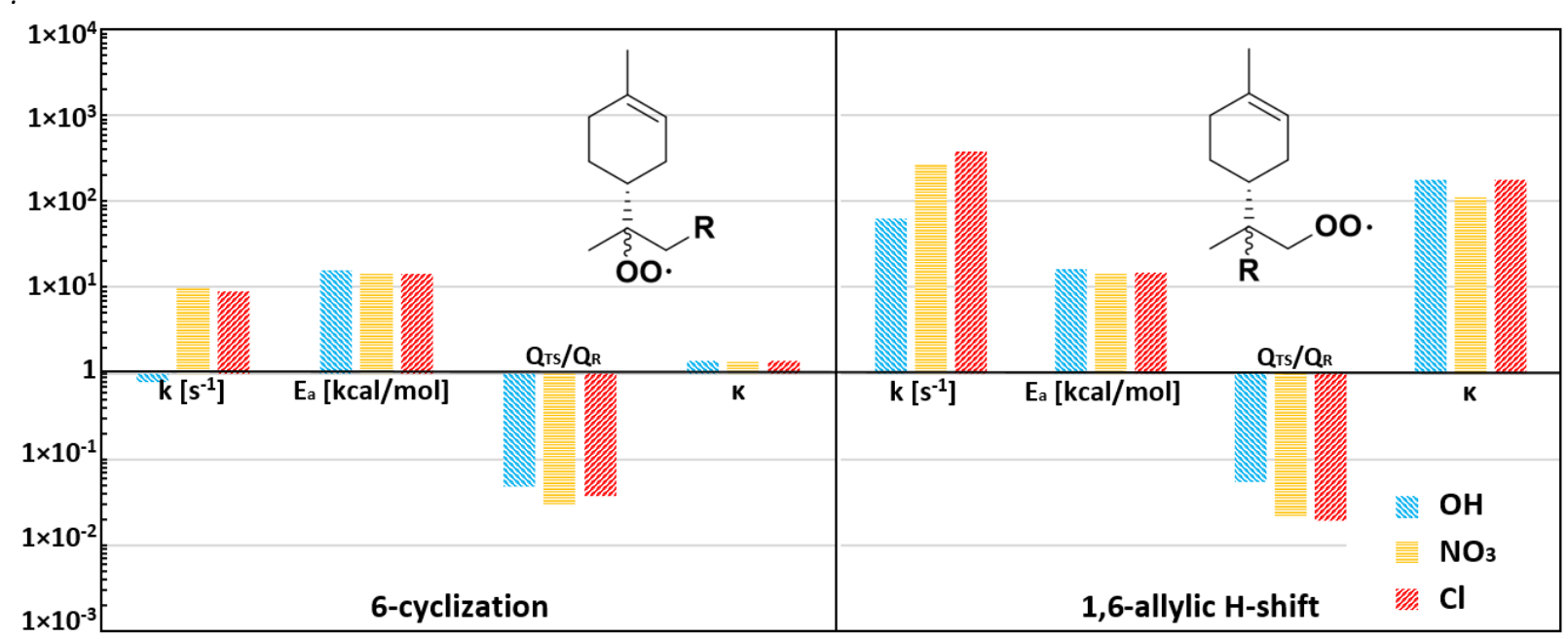

Figure S 2. Comparison of the unimolecular reaction rate coefficients $(k)$, reaction energy barriers $\left(E_{a}\right)$, ratios of summed partition functions exponentially weighted by relative conformer energy $\left(Q_{T S} / Q_{R}\right)$ and tunneling factors $(k)$ between $\mathrm{OH}, \mathrm{NO} \mathrm{O}_{3}$ and $\mathrm{Cl}$ peroxy radical ( $L a$ and $L b$ ) calculated at the $\omega B 97 X-D$ level. The functional group $R$ can be either $\mathrm{OH}, \mathrm{NO}_{3}$ or $\mathrm{Cl}$. Only the result for the ( $R, R$ ) stereoisomers are shown here. The corresponding values for the $(R, S)$ stereoisomers can be found in Table $S 7$. 
Table S 8. The lowest energy $\omega B 97 X-D$ conformers of reactant and $\mathrm{TS}$ for $\mathrm{OH}, \mathrm{NO}_{3}$ and $\mathrm{Cl}$ derived peroxy radicals $\mathrm{La}$ and $\mathrm{Lb}$. White: hydrogen. Grey: carbon. Red: Oxygen. Blue: nitrogen. Green: chlorine. For the $\mathrm{OH}$ derived La and Lb reactant conformers, the hydroxy group is directed towards the peroxy group indicating the presence of a hydrogen bond.

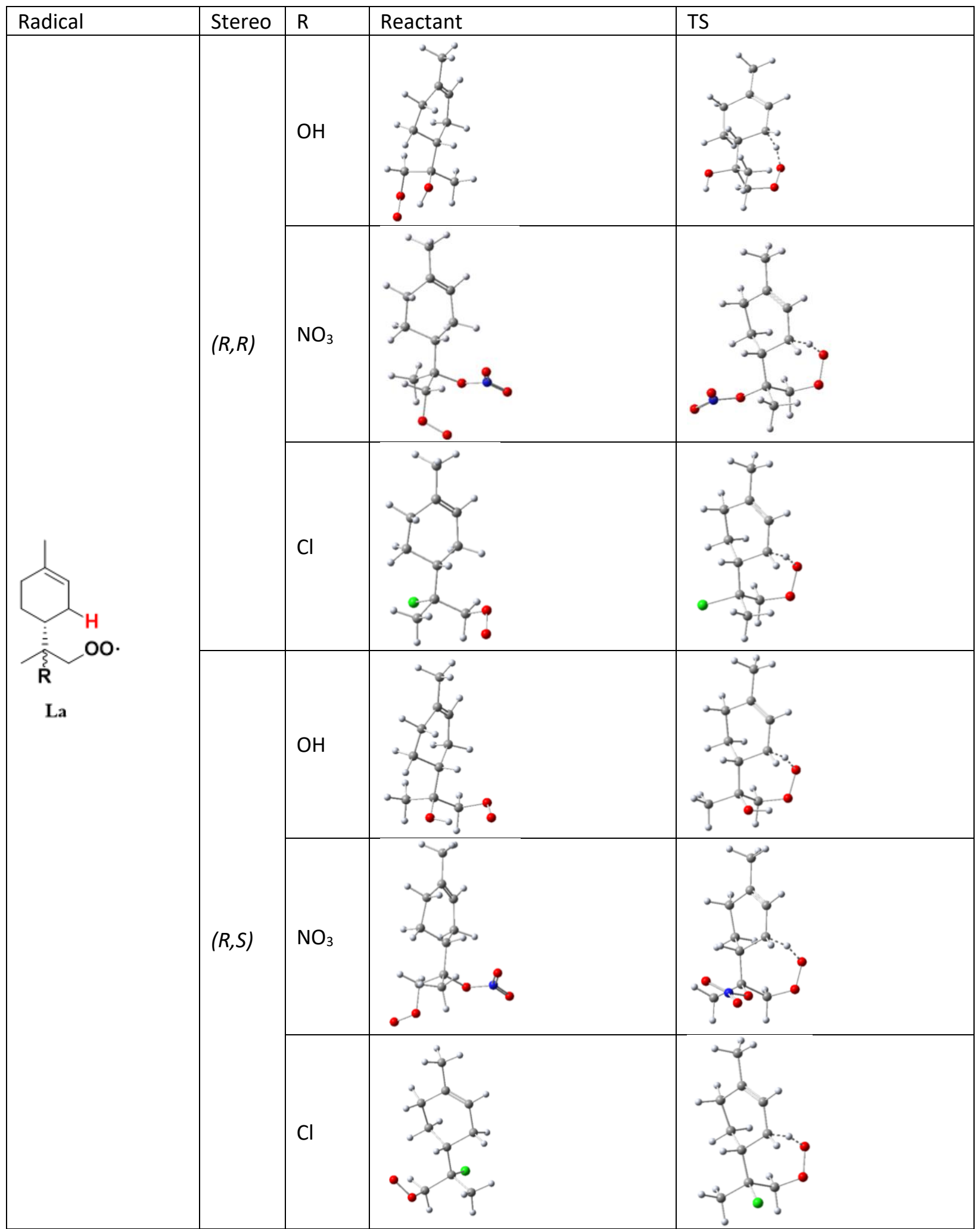




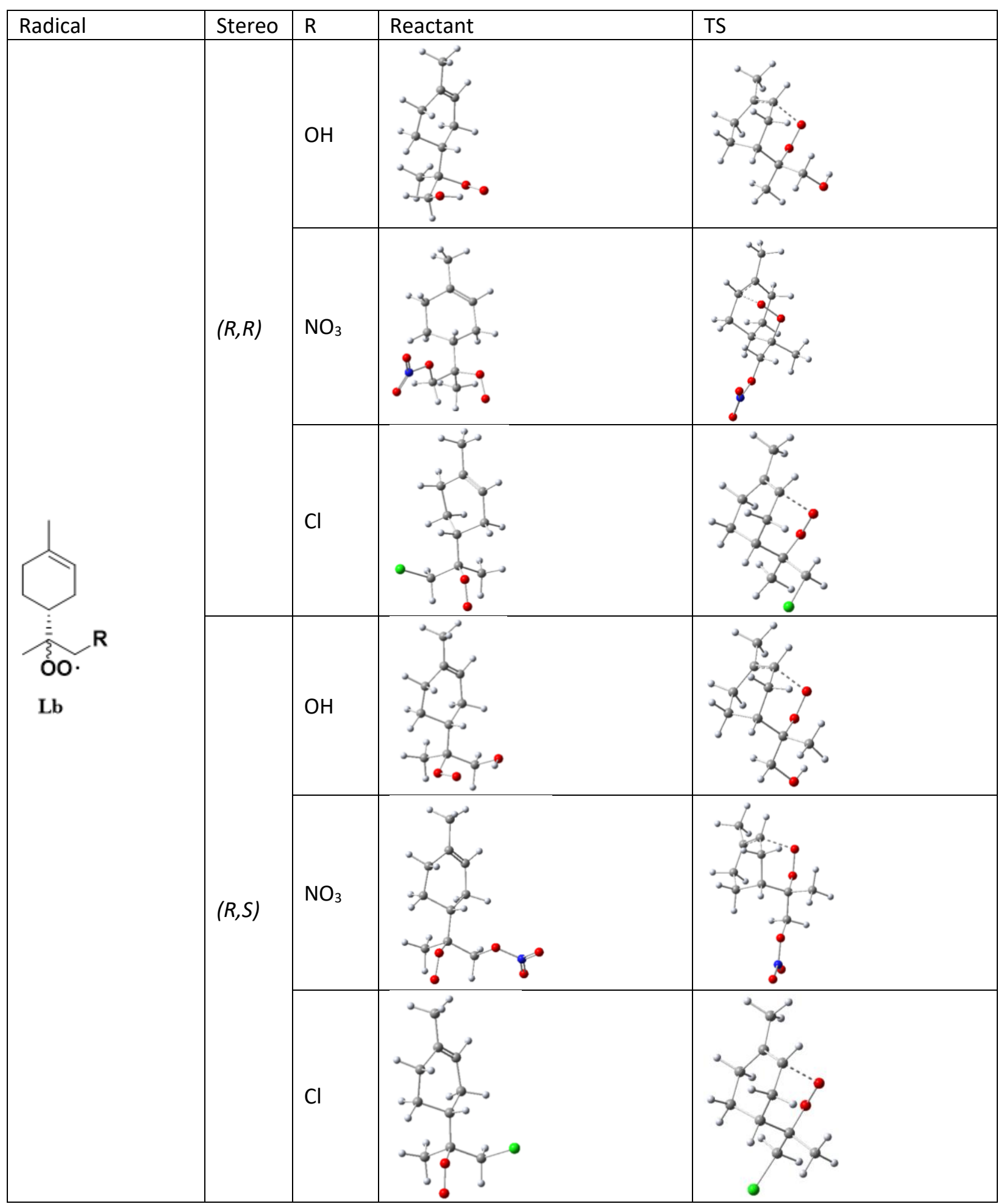




\section{S5. Proposed oxidation pathways in pristine environments}

Table S 9. The product prediction rules in pristine environments, $k_{\text {bimolecular }}^{\prime} \sim 3 \times 10^{-2} s^{-1}$.

\begin{tabular}{|c|c|}
\hline 1 & $\begin{array}{l}\text { H-shifts can happen back and forward rapidly between a hydroperoxy and a peroxy group until } \\
\text { terminated by other pathways. }{ }^{3}\end{array}$ \\
\hline 2 & $\begin{array}{l}\text { For the cyclization reactions, the } 5-, 6 \text { - and } 7 \text {-membered ring formation reactions are able to } \\
\text { compete with the possible bimolecular reactions }\left(k_{5-c y c .}>k_{6-c y c .}>k_{7-c y c .}>k^{\prime}{ }_{\text {bimolecular }}\right){ }^{4}\end{array}$ \\
\hline 3 & $\begin{array}{l}\text { For the } \mathrm{H} \text {-shift reactions, the } 1,4 \text { to } 1,9 \text {-aldehydic } \mathrm{H} \text {-shifts are considered the most competitive } \\
\text { pathways ( } \mathrm{k}_{\text {alde. }}>\mathrm{k}^{\prime} \text { bimolecular). }{ }^{2,3}\end{array}$ \\
\hline 4 & $\begin{array}{l}\text { When a hydrogen is abstracted from the aldehyde group, both the CO loss and the } \mathrm{O}_{2} \text { addition } \\
\text { pathways are considered. }\end{array}$ \\
\hline 5 & If both cyclization reactions and aldehydic $\mathrm{H}$-shifts are possible, both possibilities are considered. \\
\hline 6 & $\begin{array}{l}\text { If no aldehydic } \mathrm{H} \text {-shift nor cyclization reactions can happen, the } 1,5 \text { to } 1,9 \text {-allylic } \mathrm{H} \text {-shifts are } \\
\text { considered. }{ }^{4}\end{array}$ \\
\hline 7 & $\begin{array}{l}\text { If none of above reactions can happen, } 1,5 \text { to } 1,7-\alpha \text {-carbonyl } H \text {-shifts are considered, the } \alpha \text { - } \\
\text { carbonyl position with a hydroperoxy or endoperoxy group being preferred. }{ }^{2,3}\end{array}$ \\
\hline 8 & $\alpha$-hydroperoxy $\mathrm{H}$-shifts result in $\mathrm{O}-\mathrm{O}$ bond scission to yield a carbonyl group and $\mathrm{OH}$ recycling. ${ }^{2,3}$ \\
\hline 9 & $\begin{array}{l}\text { a-endoperoxy H-shifts (abstracting a hydrogen from a carbon connected to an endocyclic peroxy } \\
\text { group) also leads to peroxy bond scission, forming a carbonyl group and an alkoxy radical (RO). }{ }^{5}\end{array}$ \\
\hline 10 & $\alpha$-hydroxy and $\alpha$-hydroperoxy $\mathrm{H}$-shifts are considered next. ${ }^{3}$ \\
\hline 11 & $\begin{array}{l}\text { When a radical center is formed on an } \alpha \text {-hydroxy carbon in the } \beta \text {-position of a hydroperoxy group, } \\
\text { an epoxide can form along with } \mathrm{OH} \text { loss. } .56\end{array}$ \\
\hline 12 & $\begin{array}{l}\text { Three possible reaction channels for alkoxy radicals (RO) are considered: the isomerization } \\
\text { channel, the decomposition channel and the reaction with } \mathrm{O}_{2 \cdot}{ }^{7-9}\end{array}$ \\
\hline 13 & $\begin{array}{l}\text { When a hydroperoxy H-shift happens between a hydroperoxy and a hydroperoxy acid group, the } \\
\text { formation of the hydroperoxy acid is strongly preferred. }{ }^{10}\end{array}$ \\
\hline
\end{tabular}




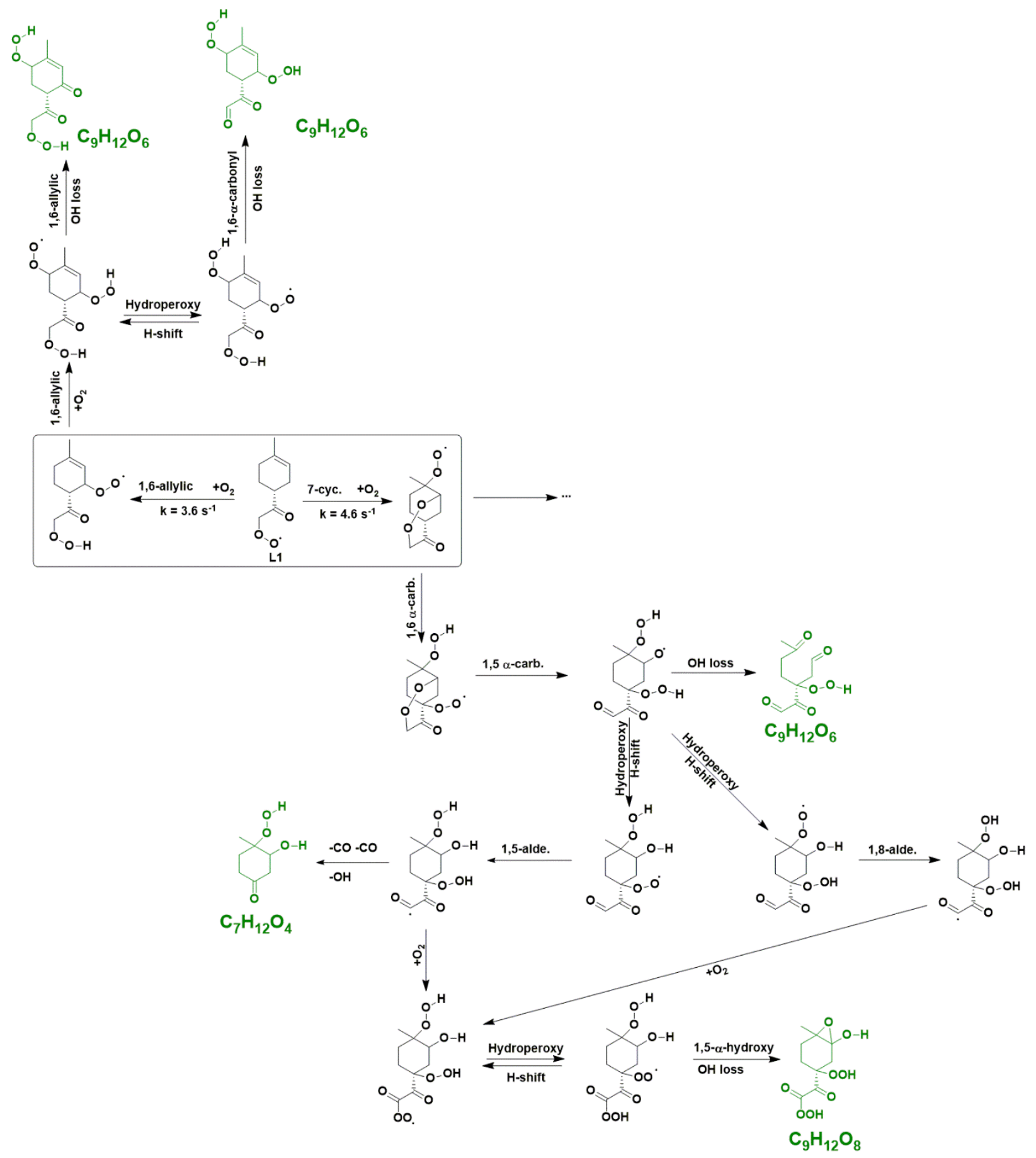

Figure S 3 . Proposed product formation pathways for L1 in pristine environments. The part in the solid box is the CCSD(T)-F12a level calculation form this work. The closed-shell final products are shown in green with their chemical formulas. 


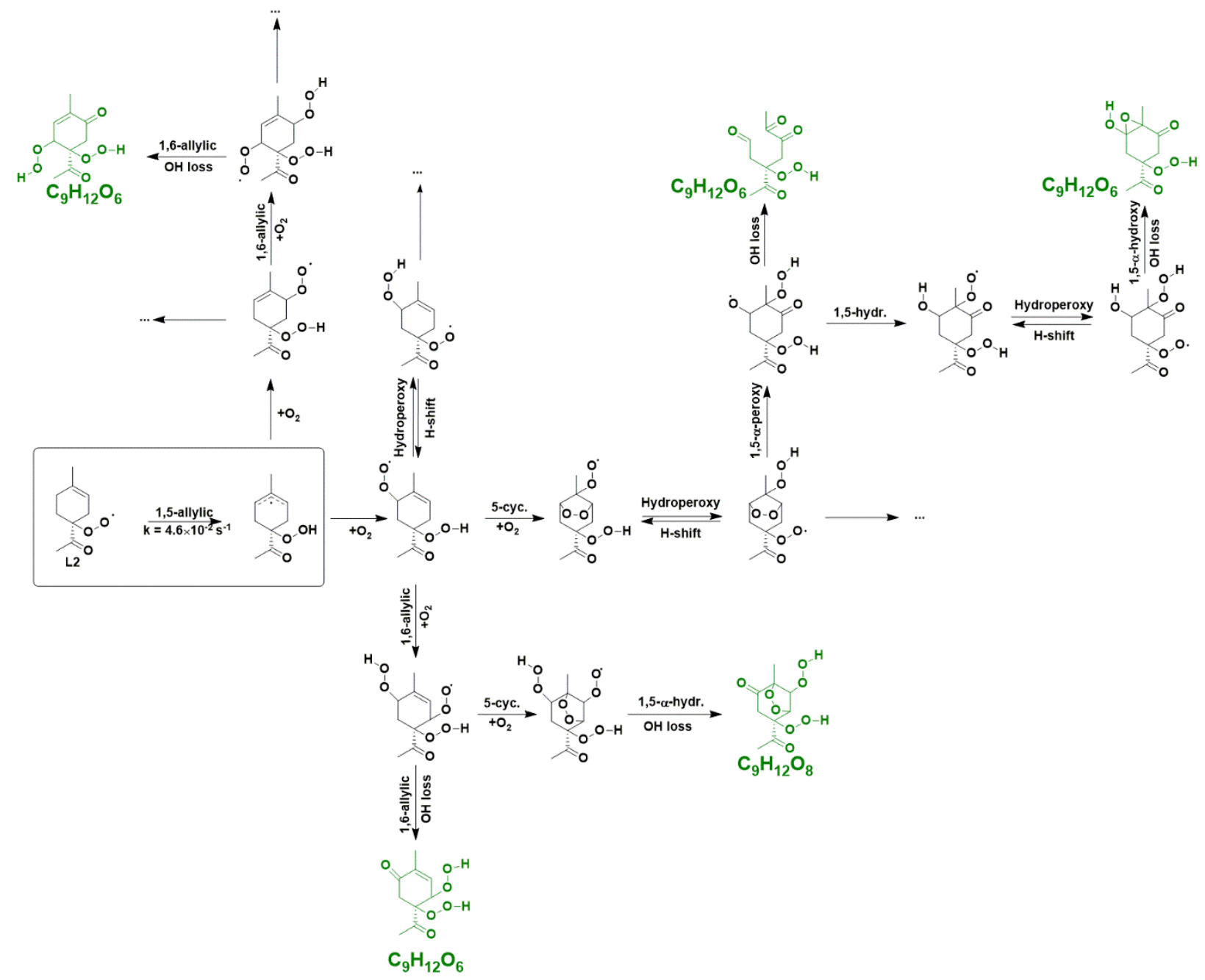

Figure S 4. Proposed product formation pathways for L2 in pristine environments. The part in the solid box is the CCSD(T)-F12a level calculation form this work. The closed-shell final products are shown in green with their chemical formulas. 


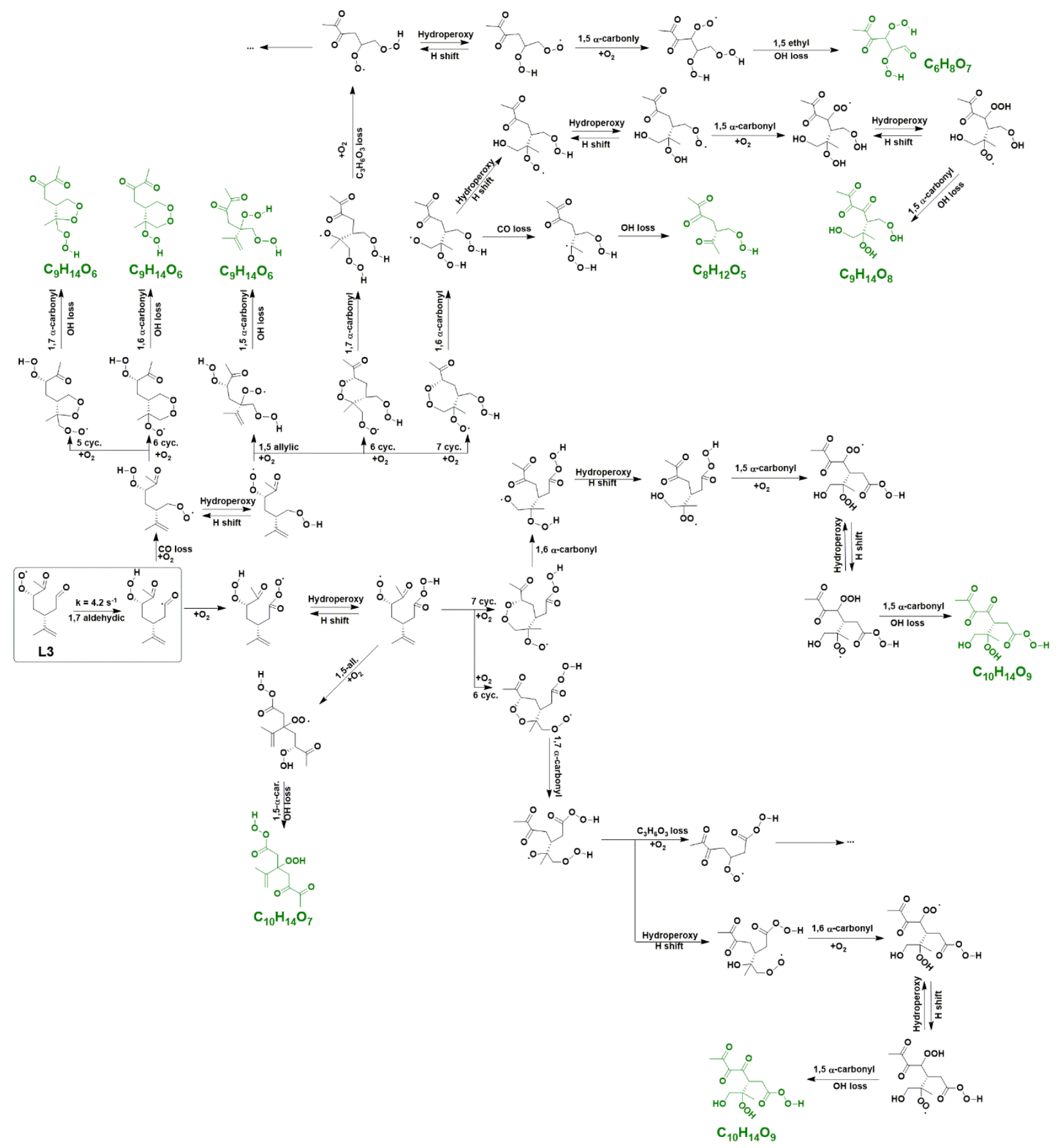

Figure S 5. Proposed product formation pathways for $(R, R)-L 3$ in pristine environments. The part in the solid box is the CCSD(T)F12a level calculation form this work. The closed-shell final products are shown in green with their chemical formulas. 


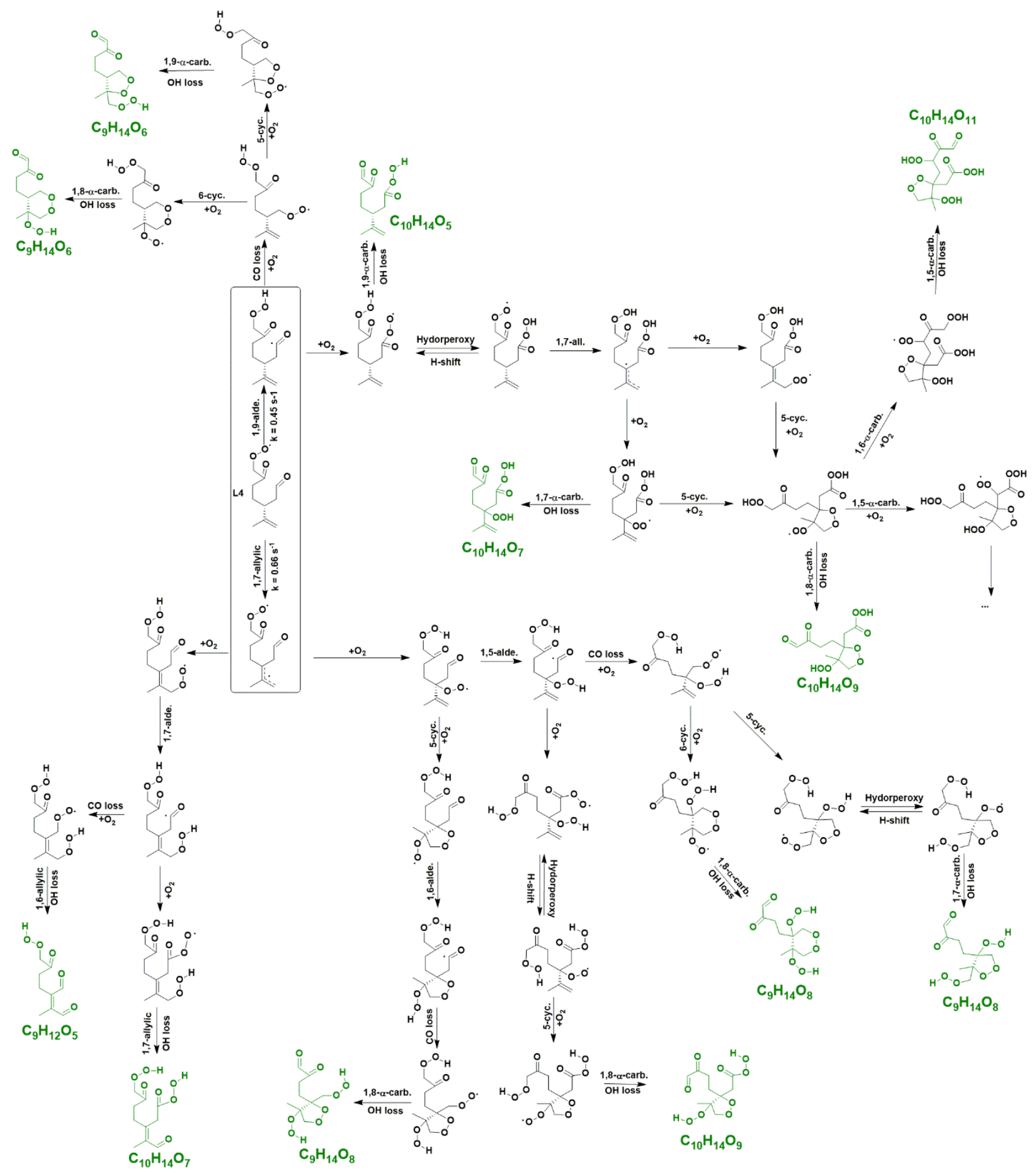

Figure S 6. Proposed product formation pathways for L4 in pristine environments. The part in the solid box is the CCSD(T)-F12a level calculation form this work. The closed-shell final products are shown in green with their chemical formulas. 


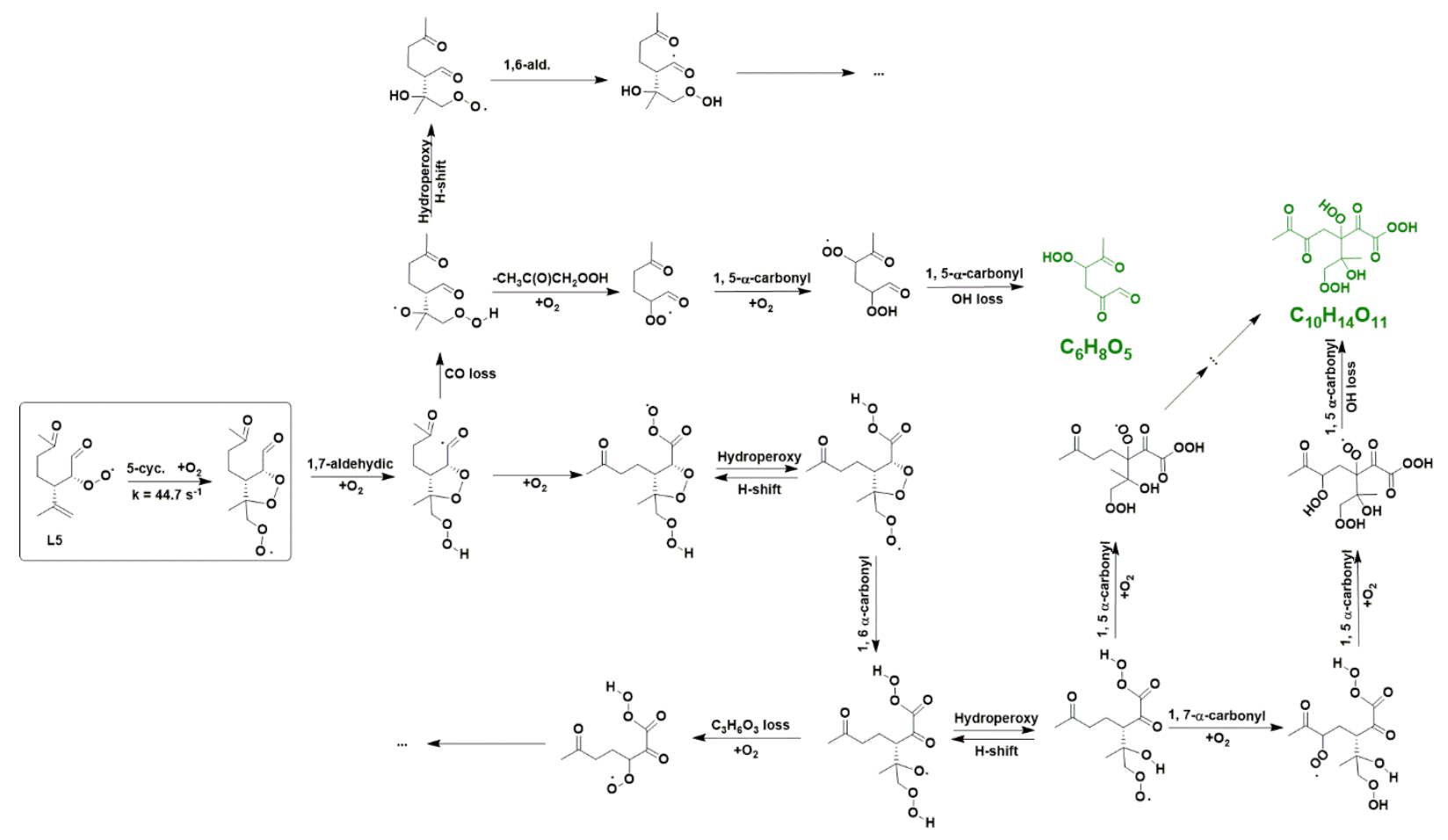

Figure S 7. Proposed product formation pathways for $(R, R)-L 5$ in pristine environments. The part in the solid box is the CCSD(T)F12a level calculation form this work. The closed-shell final products are shown in green with their chemical formulas. 


\section{S6. Proposed oxidation pathways in polluted environments}

Table S 10. The product prediction rules under polluted conditions $\left(k_{\text {bimolecular }}^{\prime} \sim 1 \mathrm{~s}^{-1}\right)$

\begin{tabular}{|c|c|}
\hline 1 & $\begin{array}{l}\text { The following reactions are considered competitive: }{ }^{4,11-13} \\
\text { - The hydroperoxy } \mathrm{H} \text {-shifts between a hydroperoxy and a peroxy group }\left(\mathrm{k}_{\text {hydrop. }}\right)^{3} \\
\text { - The 5, } 6 \text { and } 7 \text {-cyclization reaction }\left(\mathrm{k}_{5 \text {-cyc. }}>\mathrm{k}_{6 \text {-cyc. }}>\mathrm{k}_{7 \text {-cyc. }}\right)^{13} \\
\text { - The 1,4 to 1,9-aldehydic (alde.) } \mathrm{H} \text {-shifts }\left(\mathrm{k}_{6 \text {-alde. }} \approx \mathrm{k}_{\text {7-alde. }}>\mathrm{k}_{4 \text {-alde. }} \approx \mathrm{k}_{9 \text {-alde. }}\right)^{3,11} \\
\text { - The 1,6 and 1,7-allylic (all.) } \mathrm{H} \text {-shift }\left(\mathrm{k}_{6 \text {-all. }} \approx \mathrm{k}_{7 \text {-all. }}\right)^{11,14} \\
\text { - The listed reactions are considered in the order: } \mathrm{k}_{\text {hydrop. }}>\mathrm{k}_{\text {cyc. }} \approx \mathrm{k}_{\text {alde. }}>\mathrm{k}_{\text {all. }}\end{array}$ \\
\hline 2 & $\begin{array}{l}\text { Among the reactions listed in }(1) \text {, the 7-cyclization, } 1,9 \text { and } 1,4 \text {-aldehydic } \mathrm{H} \text {-shifts, 1,6 and } \\
\text { 1,7-allylic } \mathrm{H} \text {-shifts are considered to have rates comparable to the competing bimolecular } \\
\left.\text { reactions. ( } \mathrm{k}_{\text {7-cyc. }} \approx \mathrm{k}_{\text {4-alde. }} \approx \mathrm{k}_{\text {9-alde. }} \approx \mathrm{k}_{6 \text {-all. }} \approx \mathrm{k}_{\text {7-all. }} \approx \mathrm{k}_{\text {bimolecular }}\right)^{11,14}\end{array}$ \\
\hline 3 & $\begin{array}{l}\text { When more than one reaction with similar rate coefficients can happen }\left(\mathrm{k}_{1} \approx \mathrm{k}_{2}\right) \text {, all } \\
\text { possibilities are considered. }\end{array}$ \\
\hline 4 & $\begin{array}{l}\text { When a hydrogen is abstracted from an aldehyde group, both the } \mathrm{CO} \text { loss and the } \mathrm{O}_{2} \text { addition } \\
\text { pathways are considered. }\end{array}$ \\
\hline 5 & $\begin{array}{l}\alpha \text {-hydroperoxy } \mathrm{H} \text {-shifts result in } \mathrm{O}-\mathrm{O} \text { bond scission to yield a carbonyl group and } \mathrm{OH} \text { recycling. } \\
3\end{array}$ \\
\hline 6 & $\begin{array}{l}\alpha \text {-endoperoxy } \mathrm{H} \text {-shifts also lead to } \mathrm{O}-\mathrm{O} \text { bond scission to yield a carbonyl group and an alkoxy } \\
\text { radical (RO). }{ }^{15}\end{array}$ \\
\hline 7 & $\begin{array}{l}\text { Three possible reaction channels for alkoxy radical (RO) are considered: the isomerization } \\
\text { channel, the decomposition channel and the reaction with } \mathrm{O}_{2} \cdot{ }^{7-9}\end{array}$ \\
\hline 8 & $\begin{array}{l}\text { When a hydroperoxy H-shift happens between a hydroperoxy and a hydroperoxy acid group, } \\
\text { the formation of the hydroperoxy acid is strongly preferred. }{ }^{10}\end{array}$ \\
\hline
\end{tabular}




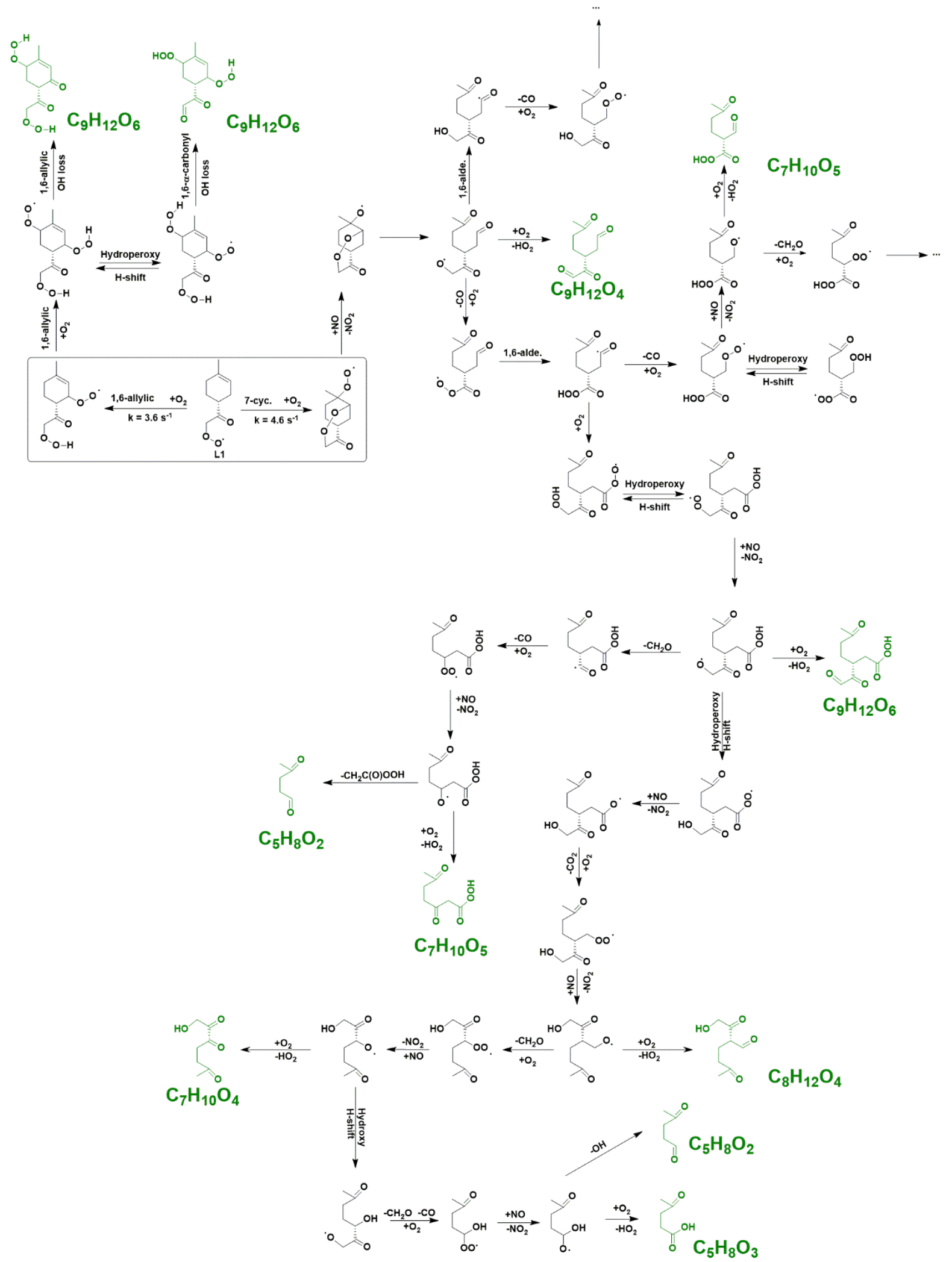

Figure S 8. Proposed product formation pathways for $L 1$ in polluted environments. The part in the solid box is the CCSD(T)-F12a level calculation form this work. The closed-shell final products are shown in green with the chemical formulas. 


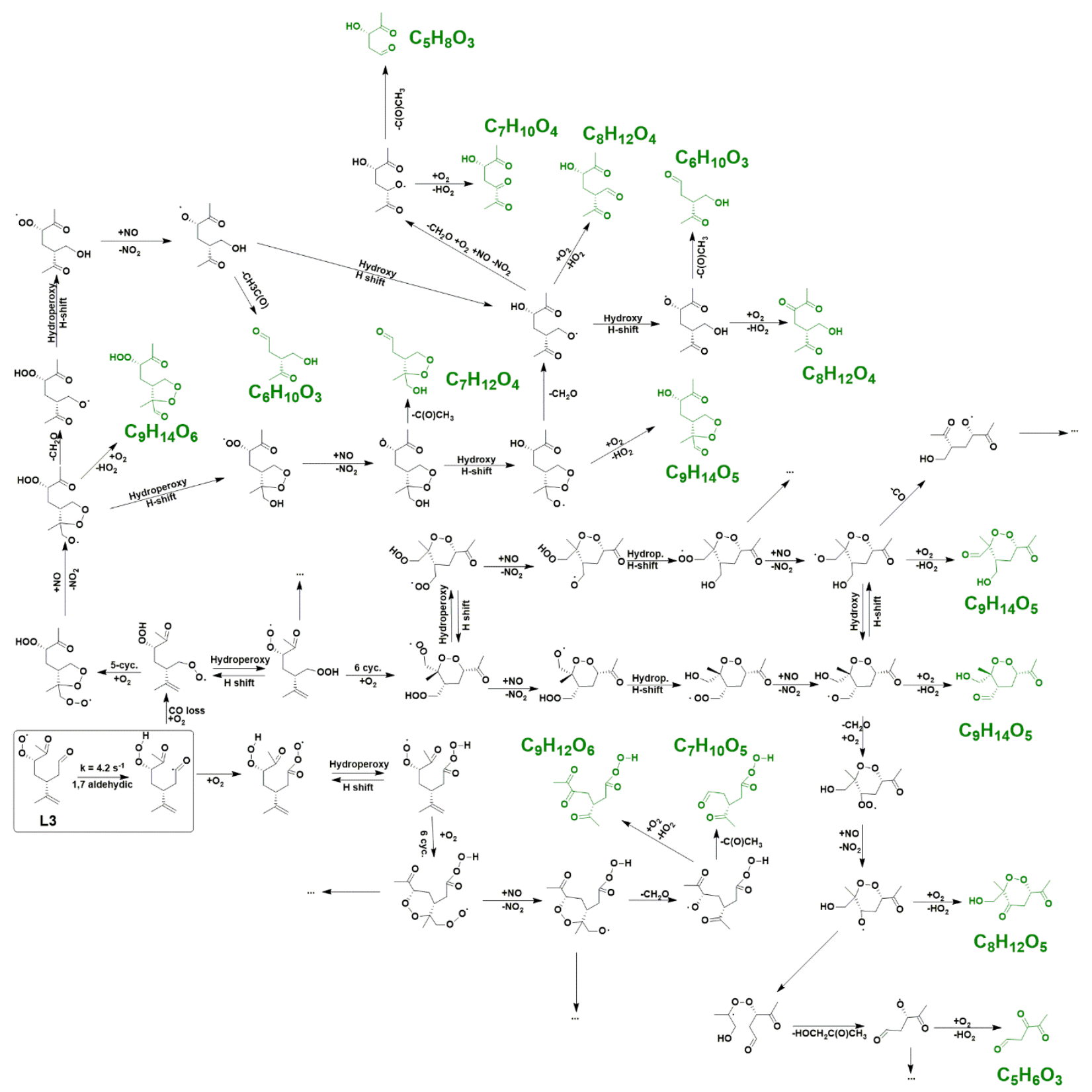

Figure S 9. Proposed product formation pathways for $(R, R)-L 3$ in polluted environments. The part in the solid box is the CCSD(T)F12a level calculation form this work. The closed-shell final products are shown in green with the chemical formulas. 


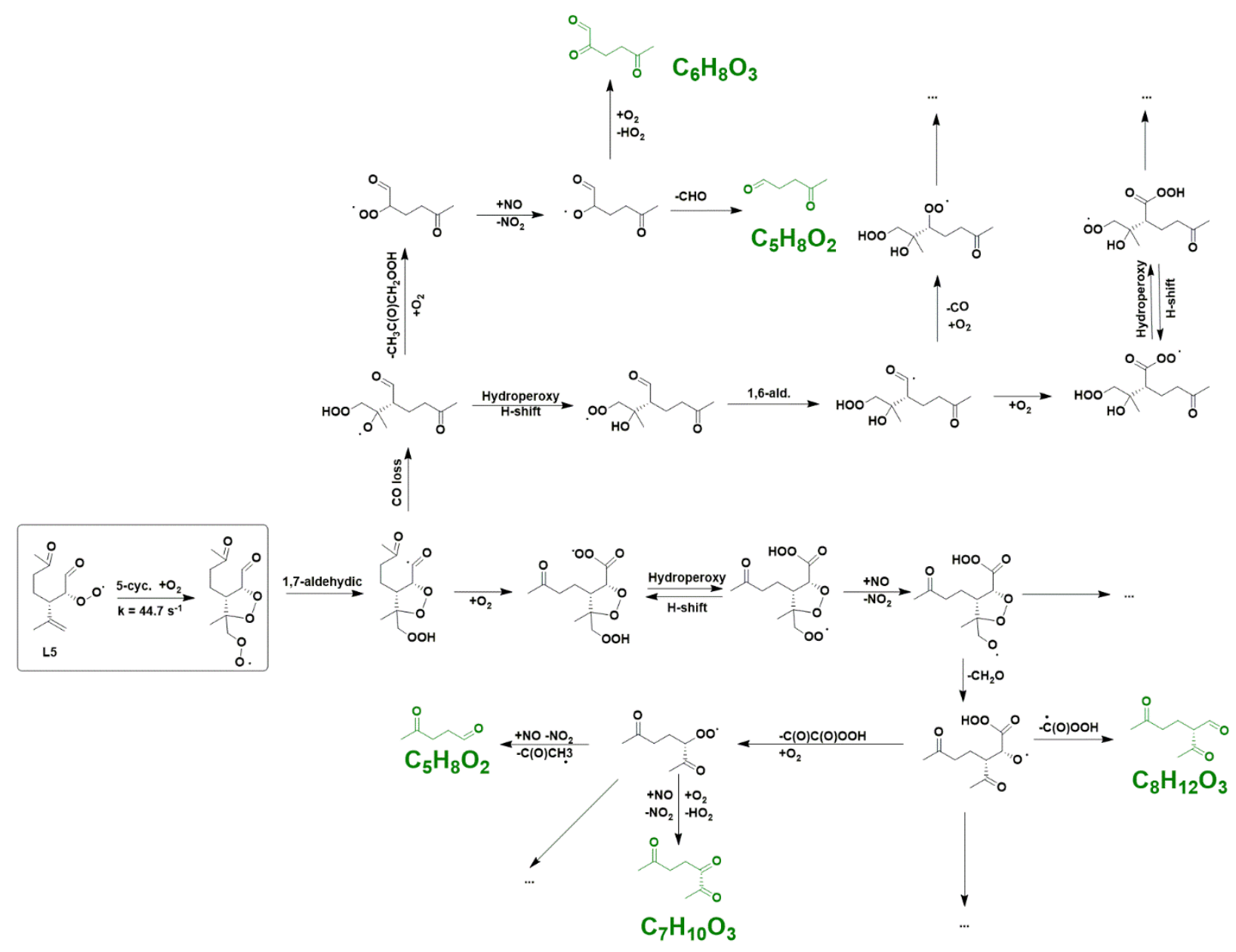

Figure S 10. Proposed product formation pathways for (S,S)-L5 in polluted environments. The part in the solid box is the CCSD(T)F12a level calculation form this work. The closed-shell final products are shown in green with the chemical formulas. 


\section{S7. Temperature dependence of the calculated reaction rate coefficients}

Table S 11. The temperature dependency of the CCSD(T)-F12a level calculated unimolecular reaction rate coefficients. Two different types of fitting methods are provided.

\begin{tabular}{|c|c|c|c|c|c|c|c|}
\hline \multirow{2}{*}{ Radical } & \multirow{2}{*}{ Fitting } & \multicolumn{3}{|c|}{$k=A \exp (-B / T) \exp \left(C / T^{3}\right)$} & \multicolumn{3}{|c|}{$k=A \exp (-E a / R T)$} \\
\hline & & $A$ & B & $\mathrm{C}$ & $\mathrm{A}\left[\mathrm{s}^{-1}\right]$ & $\mathrm{Ea} / \mathrm{R}[\mathrm{K}]$ & $\mathrm{Ea}[\mathrm{kcal} / \mathrm{mol}]$ \\
\hline \multirow{6}{*}{$\begin{array}{l}\mathrm{O}_{-} \\
\mathrm{L} \\
\mathrm{L}\end{array}$} & 1,5- $\alpha$-carbonyl & $7.10 \mathrm{E}+11$ & $1.05 \mathrm{E}+04$ & $1.55 \mathrm{E}+08$ & $1.50 \mathrm{E}+06$ & 4803.27 & 9.55 \\
\hline & 1,6-allylic & $2.14 \mathrm{E}+11$ & $9.08 \mathrm{E}+03$ & $1.49 \mathrm{E}+08$ & $7.08 \mathrm{E}+05$ & 3621.08 & 7.20 \\
\hline & 1,6-alkyl & $4.42 E+11$ & $9.97 \mathrm{E}+03$ & $6.86 \mathrm{E}+07$ & $1.34 \mathrm{E}+09$ & 7462.85 & 14.83 \\
\hline & 1,7-allylic & $1.95 \mathrm{E}+11$ & $8.91 E+03$ & $1.08 \mathrm{E}+08$ & $2.08 \mathrm{E}+07$ & 4954.77 & 9.85 \\
\hline & 7-cyclization & $7.31 \mathrm{E}+10$ & $7.06 \mathrm{E}+03$ & $4.95 \mathrm{E}+06$ & $4.81 \mathrm{E}+10$ & 6879.50 & 13.67 \\
\hline & 8-cyclization & $4.37 E+10$ & $8.69 E+03$ & $4.97 E+06$ & $2.872 E+10$ & 8511.39 & 16.91 \\
\hline \multirow{4}{*}{ L2 } & 1,4-allylic & $3.77 E+12$ & $1.34 E+04$ & $2.46 \mathrm{E}+08$ & $3.66 \mathrm{E}+03$ & 4457.62 & 8.86 \\
\hline & 1,5-allylic & $2.46 \mathrm{E}+12$ & $1.12 E+04$ & $1.61 E+08$ & $3.07 E+06$ & 5360.27 & 10.65 \\
\hline & 5-cyclization & $2.20 \mathrm{E}+12$ & $1.00 \mathrm{E}+04$ & $7.78 \mathrm{E}+06$ & $1.14 \mathrm{E}+12$ & 9732.87 & 19.34 \\
\hline & 6-cyclization & $1.64 \mathrm{E}+12$ & $1.03 E+04$ & $6.47 E+06$ & $9.51 E+11$ & 10061.23 & 19.99 \\
\hline \multirow{6}{*}{$\underbrace{(1}_{(R, R)-\mathrm{L}}$} & 1,5-allylic & 5.75E-04 & $6.81 E+03$ & $9.07 E+07$ & $2.73 \mathrm{E}-07$ & 3497.53 & 6.95 \\
\hline & 1,6-a-carbonyl & 7.33E-04 & $8.08 E+03$ & $1.61 E+08$ & 8.87E-10 & 2180.55 & 4.33 \\
\hline & 1,7-aldehydic & $3.53 \mathrm{E}-04$ & $5.66 \mathrm{E}+03$ & $7.41 E+07$ & $6.82 \mathrm{E}-07$ & 2956.20 & 5.87 \\
\hline & 1,7-allylic & $1.96 \mathrm{E}-04$ & $6.32 E+03$ & $1.04 \mathrm{E}+08$ & $3.06 \mathrm{E}-08$ & 2526.24 & 5.02 \\
\hline & 6-cyclization & $4.45 \mathrm{E}-05$ & $4.30 E+03$ & $5.77 E+06$ & $2.73 \mathrm{E}-05$ & 4086.88 & 8.12 \\
\hline & 7-cyclization & $5.46 \mathrm{E}-04$ & $5.56 \mathrm{E}+03$ & $4.86 E+06$ & $3.62 \mathrm{E}-04$ & 5383.66 & 10.70 \\
\hline \multirow{7}{*}{$\underbrace{\mathrm{L}_{1}^{0}}_{(R, S)-\mathrm{L} 3}$} & 1,5-allylic & $3.14 \mathrm{E}+11$ & $9.11 E+03$ & $1.05 E+08$ & $4.40 E+07$ & 5267.81 & 10.47 \\
\hline & 1,6- $\alpha$-carbonyl & $1.03 E+11$ & $9.99 E+03$ & $1.21 \mathrm{E}+08$ & $3.89 E+06$ & 5576.37 & 11.08 \\
\hline & 1,7-aldehydic & $1.55 E+10$ & $7.25 \mathrm{E}+03$ & $8.55 E+07$ & $1.14 \mathrm{E}+07$ & 4131.25 & 8.21 \\
\hline & 1,7-allylic & $2.99 \mathrm{E}+10$ & $7.86 \mathrm{E}+03$ & $1.10 E+08$ & $2.74 E+06$ & 3834.42 & 7.62 \\
\hline & 6-cyclization & $2.97 E+10$ & $6.80 E+03$ & $6.28 \mathrm{E}+06$ & $1.75 E+10$ & 6568.498 & 13.05 \\
\hline & 7-cyclization & $9.86 E+10$ & $7.81 E+03$ & $5.45 E+06$ & $6.22 \mathrm{E}+10$ & 7609.33 & 15.12 \\
\hline & 1,7-allylic & $3.52 \mathrm{E}+10$ & $8.65 E+03$ & $1.21 E+08$ & $1.27 \mathrm{E}+06$ & 4217.46 & 8.38 \\
\hline
\end{tabular}




\begin{tabular}{|c|c|c|c|c|c|c|c|}
\hline \multirow{4}{*}{\}$^{0}$} & 1,8- $\alpha$-carbonyl & $1.86 \mathrm{E}+10$ & $1.05 E+04$ & $1.46 \mathrm{E}+08$ & $8.02 E+04$ & 5181.53 & 10.30 \\
\hline & 1,9-aldehydic & $6.70 E+09$ & $7.94 \mathrm{E}+03$ & $9.21 \mathrm{E}+07$ & $2.83 E+06$ & 4573.79 & 9.09 \\
\hline & 1,9-allylic & $2.87 \mathrm{E}+10$ & $9.72 E+03$ & $1.40 \mathrm{E}+08$ & $2.12 \mathrm{E}+05$ & 4605.46 & 9.15 \\
\hline & 9-cyclization & $3.53 E+09$ & $7.55 E+03$ & $6.87 \mathrm{E}+06$ & $1.98 \mathrm{E}+09$ & 7297.01 & 14.50 \\
\hline \multirow{6}{*}{$\underbrace{O}_{(S, S)-L 5}$} & 1,4-aldehydic & $1.48 \mathrm{E}+12$ & $9.72 \mathrm{E}+03$ & $1.36 \mathrm{E}+08$ & $1.59 \mathrm{E}+07$ & 4770.84 & 9.48 \\
\hline & 1,5-alkyl & $1.41 \mathrm{E}+12$ & $1.12 \mathrm{E}+04$ & $7.05 E+07$ & $3.67 E+09$ & 8629.63 & 17.15 \\
\hline & 1,6-allylic & $1.92 \mathrm{E}+11$ & $1.00 E+04$ & $1.37 \mathrm{E}+08$ & $1.75 E+06$ & 4988.00 & 9.91 \\
\hline & 1,6 - $\alpha$-carbonyl & $6.62 E+10$ & $1.03 E+04$ & $1.62 E+08$ & $7.69 E+04$ & 4356.80 & 8.66 \\
\hline & 5-cyclization & $1.67 \mathrm{E}+11$ & $5.84 \mathrm{E}+03$ & $5.56 \mathrm{E}+06$ & $1.04 \mathrm{E}+11$ & 5639.51 & 11.21 \\
\hline & 6-cyclization & $9.33 E+10$ & $6.41 \mathrm{E}+03$ & $6.27 E+06$ & $5.50 \mathrm{E}+10$ & 6178.00 & 12.28 \\
\hline \multirow{6}{*}{ 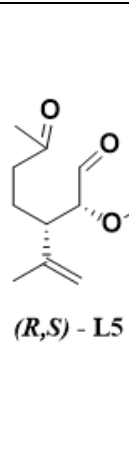 } & 1,4-aldehydic & $4.23 E+12$ & $1.01 E+04$ & $1.30 \mathrm{E}+08$ & $7.26 \mathrm{E}+07$ & 5382.09 & 10.70 \\
\hline & 1,5-alkyl & $1.05 \mathrm{E}+12$ & $1.02 \mathrm{E}+04$ & $6.92 \mathrm{E}+07$ & $3.03 E+09$ & 7688.00 & 15.28 \\
\hline & 1,6-allylic & $2.37 E+11$ & $1.02 \mathrm{E}+04$ & $1.37 \mathrm{E}+08$ & $2.16 \mathrm{E}+06$ & 5195.88 & 10.33 \\
\hline & 1,6- $\alpha$-carbonyl & $6.26 \mathrm{E}+11$ & $1.03 E+04$ & $1.43 \mathrm{E}+08$ & $3.58 \mathrm{E}+06$ & 5093.84 & 10.12 \\
\hline & 5-cyclization & $7.15 E+10$ & $6.18 \mathrm{E}+03$ & $6.46 \mathrm{E}+06$ & $4.14 \mathrm{E}+10$ & 5943.67 & 11.81 \\
\hline & 6-cyclization & $3.14 \mathrm{E}+11$ & $7.66 \mathrm{E}+03$ & $6.09 \mathrm{E}+06$ & $1.88 \mathrm{E}+11$ & 7440.05 & 14.78 \\
\hline
\end{tabular}




\section{References}

1. Møller, K. H.; Otkjær, R. V.; Hyttinen, N.; Kurten, T.; Kjaergaard, H. G., Cost-Effective Implementation of Multiconformer Transition State Theory for Peroxy Radical Hydrogen Shift Reactions. J. Phys. Chem. A 2016, 120, 10072-10087.

2. Vereecken, L.; Nozière, B., H Migration in Peroxy Radicals under Atmospheric Conditions. Atmos. Chem. Phys. 2020, 20, 7429-7458.

3. Møller, K. H. ${ }^{+}$, Bates K. H. ${ }^{\ddagger}$, and Kjaergaard H. G. ${ }^{\dagger}$, The Importance of Peroxy Radical HydrogenShift Reactions in Atmospheric Isoprene Oxidation. J. Phys. Chem. A 2019, 123, 920-932.

4. M Mller, K. H.; Otkjær, R. V.; Chen, J.; Kjaergaard, H. G., Double Bonds Are Key to Fast Unimolecular Reactivity in First-Generation Monoterpene Hydroxy Peroxy Radicals. J. Phys. Chem. A 2020, 124, 28852896.

5. Wang, L.; Wu, R.; Xu, C., Atmospheric Oxidation Mechanism of Benzene. Fates of Alkoxy Radical Intermediates and Revised Mechanism. J. Phys. Chem. A 2013, 117, 14163-8.

6. Møller, K. H.; Kurtén, T.; Bates, K. H.; Thornton, J. A.; Kjaergaard, H. G., Thermalized Epoxide Formation in the Atmosphere. J. Phys. Chem. A 2019, 123, 10620-10630.

7. Atkinson, R., Atmospheric Reactions of Alkoxy and Hydroxyalkoxy Radicals. Int. J. Chem. Kinet. 1997, 29, 99-111.

8. Vereecken, L.; Peeters, J., A Structure-Activity Relationship for the Rate Coefficient of H-Migration in Substituted Alkoxy Radicals. Phys. Chem. Chem. Phys. 2010, 12, 12608-20.

9. Peeters J., Fantechi G., Vereecken, L., A Generalized Structure-Activity Relationship for the Decomposition of (Substituted) Alkoxy Radicals. J. Atmos. Chem. 2004, 48, 59-80.

10. Knap, H. C.; Jorgensen, S., Rapid Hydrogen Shift Reactions in Acyl Peroxy Radicals. J. Phys. Chem. A 2017, 121, 1470-1479.

11. Vereecken, L.; Nozière, B., H migration in peroxy radicals under atmospheric conditions. Atmos. Chem. Phys. 2020, 20, 7429-7458.

12. Xu, L.; Møller, K. H.; Crounse, J. D.; Otkjær, R. V.; Kjaergaard, H. G.; Wennberg, P. O., Unimolecular Reactions of Peroxy Radicals Formed in the Oxidation of $\alpha$-Pinene and $\beta$-Pinene by Hydroxyl Radicals. J. Phys. Chem. A 2019, 123, 1661-1674.

13. Peeters J., Vereecken, L.., Nontraditional (Per)oxy Ring-Closure Paths in the Atmospheric Oxidation of Isoprene and Monoterpenes. J. Phys. Chem. A 2004, 108, 5197-5204.

14. Møller, K. H.; Otkjær, R. V.; Chen, J.; Kjaergaard, H. G., Double Bonds Are Key to Fast Unimolecular Reactivity in First-Generation Monoterpene Hydroxy Peroxy Radicals. J. Phys. Chem. A 2020, 124, 28852896.

15. Wang, L.; Wu, R.; Xu, C., Atmospheric oxidation mechanism of benzene. Fates of alkoxy radical intermediates and revised mechanism. J. Phys. Chem. A 2013, 117, 14163-8. 